\title{
Temporal Integrators for Fluctuating Hydrodynamics
}

\author{
Steven Delong, ${ }^{1}$ Boyce E. Griffith, ${ }^{2}$ Eric Vanden-Eijnden, ${ }^{1,}$ 米 and Aleksandar Donev ${ }^{1,}$ † \\ ${ }^{1}$ Courant Institute of Mathematical Sciences, New York University, New York, NY 10012 \\ ${ }^{2}$ Leon H. Charney Division of Cardiology, Department of Medicine, \\ New York University School of Medicine, New York, NY 10016
}

\begin{abstract}
Including the effect of thermal fluctuations in traditional computational fluid dynamics requires developing numerical techniques for solving the stochastic partial differential equations of fluctuating hydrodynamics. These Langevin equations possess a special fluctuation-dissipation structure that needs to be preserved by spatio-temporal discretizations in order for the computed solution to reproduce the correct long-time behavior. In particular, numerical solutions should approximate the Gibbs-Boltzmann equilibrium distribution, and ideally this will hold even for large time step sizes. We describe finite-volume spatial discretizations for the fluctuating Burgers and fluctuating incompressible Navier-Stokes equations that obey a discrete fluctuation-dissipation balance principle just like the continuum equations. We develop implicit-explicit predictor-corrector temporal integrators for the resulting stochastic method-of-lines discretization. These stochastic Runge-Kutta schemes treat diffusion implicitly and advection explicitly, are weakly second-order accurate for additive noise for small time steps, and give a good approximation to the equilibrium distribution even for very strong fluctuations. Numerical results demonstrate that a midpoint predictor-corrector scheme is very robust over a broad range of time step sizes.
\end{abstract}

\section{INTRODUCTION}

Modeling the effects of thermal fluctuations in spatially-extended systems requires introducing Langevin stochastic forcing terms in traditional deterministic models 1, 2. Stochastic effects arise in fluid dynamics because of the random thermal motion of the molecules comprising the fluid at the microscopic level. Stochastic effects are important in flows at micro and nano scales typical of new nano- and micro-fluidic and microelectromechanical devices [3, 4, novel materials such as nanofluids [5], and biological systems such as lipid membranes, Brownian molecular motors, and nanopores [6]. Thermal fluctuations can be amplified by non-equilibrium effects and affect the macroscale, as in fluid mixing [7, 8], propagation of fronts [9, 10, combustion of lean flames, capillaries [11, 12, and hydrodynamic instabilities [13 15]. Because they span the whole range of scales from the microscopic to the macroscopic [7, 8], fluctuations need to be consistently included in all levels of description, including continuum descriptions.

Thermal fluctuations can be included in the classical Navier-Stokes-Fourier equations of fluid dynamics and related conservation laws through stochastic forcing terms, as first proposed by Landau and Lifshitz. The original formulation was for compressible single-component fluids [16. However, the methodology can be extended to other systems such as fluid mixtures [17, chemically reactive systems [18, magnetic materials [19, and others [20]. The structure of the equations of fluctuating hydrodynamics can be, to some extent, justified on the basis of the Mori-Zwanzig formalism [21, 22]. The basic idea is to add a stochastic flux corresponding to each dissipative (irreversible, diffusive) flux [2], leading to a continuum Langevin model that ensures the correct equilibrium distribution. Specifically, statistical mechanics tells us that the stationary (invariant) distribution at thermodynamic equilibrium is the Einstein distribution for isolated systems, and the Gibbs-Boltzmann distribution for systems in contact with a thermal bath.

As model equations, here we consider the fluctuating Burgers equation in one dimension and the fluctuating NavierStokes equation in two and three dimensions. These stochastic conservation laws have non-dissipative (skew-adjoint) advective terms and dissipative (self-adjoint) viscous terms, as well as stochastic forcing terms that are in fluctuationdissipation balance with the dissipative terms. Spatial discretizations of the corresponding continuum (functional) operators should preserve these (anti-)symmetry properties in order to obey a discrete fluctuation-dissipation balance. In general, constructing such spatial discretizations (coarse-grained Langevin models) is non-trivial and may be at odds with other considerations such as deterministic stability. For example, upwind discretizations commonly used for advection-diffusion equations add artificial dissipation to the equations, thus violating fluctuation-dissipation balance.

Based on prior work by us and others, we construct spatial discretizations for the fluctuating Burgers and fluctuating incompressible Navier-Stokes equations that obey a discrete fluctuation-dissipation balance principle just like the

*Electronic address: eve2@courant.nyu.edu

†Electronic address: donev@courant.nyu.edu 
continuum equations. The main challenge is in constructing temporal integrators for the resulting large-scale system of stochastic differential equations. Ideally, the temporal integrators should have higher-order short-time accuracy but also lead to long-time dynamics that is in agreement with fluctuation-dissipation balance. Generalizing temporal integrators that are favored for Langevin equations in low-dimensional systems (e.g., molecular dynamics) 23 to the equations of fluctuating hydrodynamics would require implicitly handling the non-linear advective terms. Solving the resulting large-scale non-linear system of equations is computationally expensive in three dimensions for the NavierStokes equations. Mixed implicit-explicit Runge-Kutta schemes are used commonly in the deterministic method of lines, and can be extended to the stochastic setting with little effort, at least for the case of additive-noise equations 24.

We derive the conditions for second-order weak accuracy of implicit-explicit predictor-corrector schemes for additive noise, and construct several candidate schemes. These semi-implicit schemes use an implicit midpoint rule for the diffusive and stochastic terms, which has the remarkable property that it gives the correct equilibrium distribution independent of the time step size. The non-linear advective terms are handled explicitly using an Euler predictor and a trapezoidal or midpoint corrector. We explain how to incorporate the stochastic forcing term in the resulting predictor-corrector schemes, and numerically study their performance on the model fluctuating Burgers equation. The numerical results suggest that the midpoint corrector is particularly robust. We then extend the spatio-temporal discretization to handle the incompressibility constraint present in the fluctuating Navier-Stokes equations in two and three spatial dimensions. We also include a passively-advected fluctuating scalar field, as encountered in practical problems. The schemes that we develop and analyze here have already been employed in Ref. [25] to construct a robust numerical solver for fluctuating incompressible flows, and to simulate the appearance of giant concentration fluctuations in diffusively mixing fluids 7,8 .

We begin by reviewing the fluctuating Navier-Stokes equations, and summarize a rather general formulation of finite-dimensional Langevin equations that obey a fluctuation-dissipation principle. In Section [II we explain in detail how to spatially discretize the fluctuating Burgers in one dimension so as to obtain a finite-dimensional system of Langevin equations with the proper structure. We turn our attention to temporal integrators for solving the resulting large-scale structured system of stochastic differential equations in Section III In Section IIID we design several implicit-explicit stochastic Runge-Kutta schemes that are second-order weakly accurate for additive noise and maintain fluctuation-dissipation balance even for large time steps. In Section IV] we explain how the spatio-temporal discretization developed for the fluctuating Burgers equation can be generalized to the fluctuating Navier-Stokes equations with a passively-advected scalar field. The performance of the proposed schemes in the nonlinear (large fluctuation) setting is investigated in Section $\mathrm{V}$, and some concluding remarks are given in Section VI. Several technical calculations are detailed in the Appendix.

\section{A. Fluctuating Hydrodynamics}

The prototype stochastic partial differential equation (SPDE) of fluctuating hydrodynamics is the fluctuating NavierStokes equation. This equation approximates the dynamics of the velocity field $\boldsymbol{v}(\boldsymbol{r}, t)$ of a simple Newtonian fluid in the isothermal and incompressible approximation, $\boldsymbol{\nabla} \cdot \boldsymbol{v}=0$,

$$
\rho\left(\partial_{t} \boldsymbol{v}+\boldsymbol{v} \cdot \boldsymbol{\nabla} \boldsymbol{v}\right)=-\boldsymbol{\nabla} \pi+\eta \boldsymbol{\nabla}^{2} \boldsymbol{v}+\boldsymbol{\nabla} \cdot\left[\left(2 k_{B} T \eta\right)^{\frac{1}{2}} \mathcal{Z}\right]+\boldsymbol{f}
$$

where $\pi$ is the non-thermodynamic pressure, $\rho$ is the (constant) density, $\eta=\rho \nu$ is the (constant) shear viscosity and $\nu$ is the kinematic viscosity, and $\boldsymbol{f}(\boldsymbol{r}, t)$ is an additional force density such as gravity [17. Note that we prefer to use the standard physics notation instead of the differential notation more common in the mathematics literature, since the noise is additive and there is no difference between the different interpretation of stochastic integrals (e.g., Ito vs. Stratonovich). In the momentum conservation law (1), the stochastic momentum flux is modeled using a white-noise random Gaussian tensor field $\mathcal{Z}(\boldsymbol{r}, t)$, that is, a tensor field whose components are independent (space-time) white noise processes,

$$
\left\langle\mathcal{Z}_{i j}(\boldsymbol{r}, t) \mathcal{Z}_{k l}\left(\boldsymbol{r}^{\prime}, t^{\prime}\right)\right\rangle=\left(\delta_{i k} \delta_{j l}\right) \delta\left(t-t^{\prime}\right) \delta\left(\boldsymbol{r}-\boldsymbol{r}^{\prime}\right)
$$

Note that in principle the stochastic momentum flux should have the symmetrized form $\left(k_{B} T \eta\right)^{1 / 2}\left(\mathcal{Z}+\mathcal{Z}^{T}\right)[17$; however, for incompressible flow with constant viscosity this is not necessary [25].

The fluctuating Navier-Stokes equation, like other augmented Langevin equations of interest [26], obeys a fluctuation-dissipation principle, as explained more precisely in Section IB. Specifically, (1) is constructed so that, at thermodynamic equilibrium, the invariant measure (equilibrium distribution) for the fluctuating velocities with 
periodic boundaries is the Gibbs-Boltzmann distribution with a coarse-grained free energy or Hamiltonian given by the kinetic energy of the fluid, formally,

$$
P_{\mathrm{eq}}(\boldsymbol{v})=Z^{-1} \exp \left[-\frac{\int d \boldsymbol{r} \rho v^{2}}{2 k_{B} T}\right] \boldsymbol{\delta}\left(\int d \boldsymbol{r} \rho \boldsymbol{v}\right) \delta(\boldsymbol{\nabla} \cdot \boldsymbol{v}) .
$$

This is ensured by constructing the stochastic forcing term so that its covariance is proportional to the viscous dissipation operator $\eta \boldsymbol{\nabla}^{2}$. The advective operator $(\boldsymbol{v} \cdot \boldsymbol{\nabla})$, is, at least formally, Hamiltonian [27 in nature, which means that it preserves the equilibrium distribution dictated by the competition between the dissipative and the stochastic forcing terms. We argue that these well-known observations about the structure of the continuum equations should guide the construction of spatio-temporal discretizations [28.

We have formally written equation (1) as an infinite dimensional stochastic differential equation. However, the interpretation of the nonlinear term $\boldsymbol{v} \cdot \boldsymbol{\nabla} \boldsymbol{v}$ requires giving a precise meaning to products of distributions, which cannot be defined in general and requires introducing some sort of regularization. An alternative is to define a discrete hydrodynamic field directly via some form of averaging of the molecular configuration of the fluid, and to obtain directly a finite-dimensional system of stochastic ordinary differential equations (SODEs) for the discrete variables through the Mori-Zwanzig formalism [22, 29, 30]. While such an approach has certain advantages from a coarse-graining perspective, the notion of a continuum equation and the applicability of traditional methods for computational fluid dynamics is lost or at least obscured.

Here we adopt a middle ground between the "continuum" and the "discrete" approach to fluctuating hydrodynamics. Specifically, we first spatially discretize the SPDE to obtain a system of SODEs, in the spirit of the "method of lines". Our focus here is on the temporal integrators for the resulting system of SODEs. We do not consider the convergence of the numerical method as the spatial discretization is refined, as one would in deterministic fluid dynamics. Rather, we fix the spatial discretization and assume that the hydrodynamic cells are sufficiently large, specifically, that they contain, on average, sufficiently many fluid molecules $N_{p} \gg 1$. This ensures that the equilibrium fluctuations will be on the order of $O\left(N_{p}^{-1 / 2}\right)$ relative to macroscopic fields. We also assume that the transport coefficients have been renormalized to account for the finite number of fluid particles (molecules) used to define the hydrodynamic fields [8, 31. There is strong numerical evidence that under these conditions spatio-temporal discretizations can correctly capture the leading-order (measurable) effects of fluctuations at large scales, such as fluctuation-driven transport in non-equilibrium systems [ [], large-scale inhomogeneities arising during free fluid mixing [25], and diffusive effects on the very long-time dynamics such as drifts in propagating fronts 32 and shocks [33].

\section{B. Fluctuation-Dissipation Balance in Generic Langevin Equations}

The fluctuating hydrodynamic formalism finds its foundation in the theory of coarse-graining [34. One of the central objects in the theory is the coarse-grained Hamiltonian or a coarse-grained free energy, which determines the Gibbs-Boltzmann equilibrium probability density for the coarse variables $\boldsymbol{x} \in \mathbb{R}^{N}$,

$$
P_{\mathrm{eq}}(\boldsymbol{x})=Z^{-1} \exp \left[-\frac{H(\boldsymbol{x})}{k_{B} T}\right]
$$

where $Z$ is a normalization factor and the scaled temperature $k_{B} T$ sets the unit of energy. We will set $k_{B} T=1$ for simplicity through the remainder of this paper. Note that in many cases the actual Hamiltonian (total energy) may be expressible in terms of the coarse-grained variables and is strictly conserved [2, but this is not the case for isothermal systems.

As discussed at length by Grabert [35, a Markovian approximation within the Mori-Zwanzig formalism can be used to obtain a generic Langevin equation of motion [26] for an arbitrary choice of the microscopic ensemble,

$$
\partial_{t} \boldsymbol{x}=-\boldsymbol{N}(\boldsymbol{x}) \frac{\partial H}{\partial \boldsymbol{x}}+\left(2 k_{B} T\right)^{1 / 2} \boldsymbol{B}(\boldsymbol{x}) \mathcal{W}(t)+\left(k_{B} T\right) \frac{\partial}{\partial \boldsymbol{x}} \cdot \boldsymbol{N}^{\star}(\boldsymbol{x}),
$$

where $\mathcal{W} \in \mathbb{R}^{N_{w}}$ denotes white noise, the formal temporal derivative of a collection of independent Brownian motions, and an Ito interpretation is assumed. We will typically suppress the explicit dependence on $\boldsymbol{x}$ and write the mobility operator as $\boldsymbol{N} \equiv \boldsymbol{N}(\boldsymbol{x})$. In the generic Langevin equation (3), the skew-adjoint operator

$$
\boldsymbol{S}=-\boldsymbol{S}^{\star}=\frac{1}{2}\left(\boldsymbol{N}^{\star}-\boldsymbol{N}\right)
$$


generates the "conservative" part of the dynamics, and the self-adjoint positive semi-definite operator

$$
\boldsymbol{M}=\boldsymbol{M}^{\star}=\frac{1}{2}\left(\boldsymbol{N}+\boldsymbol{N}^{\star}\right) \succeq \mathbf{0}
$$

generates the "dissipative" part of the dynamics, since

$$
\frac{d H}{d t}=-\left(\frac{\partial H}{\partial \boldsymbol{x}}\right)^{T} \boldsymbol{N} \frac{\partial H}{\partial \boldsymbol{x}}=-\operatorname{Re}\left[\left(\frac{\partial H}{\partial \boldsymbol{x}}\right)^{T} \cdot \boldsymbol{M} \cdot \frac{\partial H}{\partial \boldsymbol{x}}\right] \leq 0 .
$$

The operator $\boldsymbol{B}(\boldsymbol{x})$ is constrained, but not uniquely-determined, by the fluctuation-dissipation balance condition

$$
B B^{\star}=M
$$

The last term in (3) is an additional "spurious" or "thermal" drift term whose form depends on the particular interpretation of the stochastic equations [36], which we take to be in the sense of Ito. In the case of the fluctuating Burgers and Navier-Stokes equations this term will vanish.

The dynamics (3) is ergodic and time reversible (under an appropriate parity transformation for the variables) with respect to the distribution (2). It is not hard to show using the corresponding Fokker-Planck Equation that (2) is an equilibrium distribution for (3), as desired, at least if one makes the nonrestrictive assumption that $\boldsymbol{M} \succ \mathbf{0}$. More precisely, the invariant measure for the coarse-grained variables is $d \mu_{\mathrm{eq}}=\mu_{\mathrm{eq}}(d \boldsymbol{x})=P_{\mathrm{eq}}(\boldsymbol{x}) d \boldsymbol{x}$. We will assume here that the dynamics is ergodic with respect to a unique equilibrium distribution, which may not be the case if $H$ ( $\boldsymbol{x}$ ) is not defined everywhere. It is important to point out that when Langevin equations of the form (3) are applied in a non-equilibrium setting, the dynamics may not be ergodic with respect to any distribution, even at steady state.

\section{FLUCTUATING BURGERS EQUATION}

The analysis and numerical solution of the incompressible Navier-Stokes equation is complicated by the presence of the incompressibility constraint. We begin our discussion by constructing a spatial discretization of the simpler unconstrained fluctuating Burgers equation for the random field $u(x, t)$,

$$
\partial_{t} u+c u \partial_{x} u=\nu \partial_{x x}^{2} u+(2 \nu)^{\frac{1}{2}} \partial_{x} \mathcal{Z}
$$

where $\nu$ is a diffusion coefficient and $c$ sets the scale for the advection speed. This equation mimics some of the properties of the fluctuating Navier-Stokes equation (1), in particular, it obeys a fluctuation-dissipation balance principle with respect to the Gibbs-Boltzmann distribution with a Hamiltonian $H=\int d x u^{2} / 2$. The fluctuating Burgers equation can also be written in conservative form

$$
\partial_{t} u=-\partial_{x}\left[c \frac{u^{2}}{2}-\nu \partial_{x} u-(2 \nu)^{\frac{1}{2}} \mathcal{Z}\right]
$$

showing that the total momentum $\int u d x$ is conserved with periodic boundary conditions. Note that here the stochastic forcing term is linear and involves the spatial derivative of white noise [37, rather than white noise itself as in the stochastic Burgers equation studied, for example, in Ref. 38. Equations of this type arise as coarse-grained models of the behavior of one dimensional lattice gases, such as the asymmetric excluded random walk model [37].

In this section we show how the fluctuating Burgers equation can be spatially discretized in a manner that leads to a generic Langevin equation of the form (3). This construction will be extended to the Navier-Stokes equations with a passively-advected scalar in Section IV. Our approach to the spatial discretization follows standard practice in deterministic fluid dynamics. Specifically, we construct the spatially-discrete system by combining locally-accurate spatial discretizations of the differential operators (e.g., gradient, divergence and Laplacian) that appear in the the SPDE. However, in addition to focusing on accuracy and stability when choosing the spatial discretization, we pay particular attention to preserving fluctuation-dissipation balance. This means that we want to obtain a system of SODEs whose structure is given in (3) and whose invariant distribution (equilibrium distribution) is a natural discretization of the Gibbs distribution dictated by equilibrium statistical mechanics.

\section{A. Continuum Fluctuating Burgers Equation}

One can, at least formally, consider a generic Langevin equation for an infinite dimensional field 2. The fluctuating Burgers equation (4) is a prototype of such an equation. In this formalism the coarse-grained Hamiltonian is a 
functional of the field and the partial derivatives should be interpreted as functional derivatives, and contractions by a field imply integrations over the spatial domain. For equation (4), the (formal) free energy functional is

$$
H[u(x, t)]=\int \frac{u^{2}}{2} d x,
$$

so that

$$
\frac{\partial H}{\partial u} \equiv \frac{\delta H[u(x, t)]}{\delta u}=u \text {. }
$$

The dissipative and fluctuating dynamics in (4) are generated by the constant operators,

$$
\boldsymbol{M}=-\nu \partial_{x x}^{2} \text { and } \boldsymbol{B}=\nu^{\frac{1}{2}} \partial_{x}
$$

which in higher dimensions become multiples of the Laplacian and divergence operators, respectively. The conservative dynamics for the Burgers equation is Hamiltonian and generated by the skew-adjoint linear operator $\boldsymbol{S}(u)$ defined through its action on a field $w(x, t)$ [27,

$$
\boldsymbol{S}(u) w=-\frac{c}{3}\left[u \partial_{x} w+\partial_{x}(u w)\right]
$$

The $\boldsymbol{v} \cdot \boldsymbol{\nabla} \boldsymbol{v}$ term in the higher-dimensional fluctuating Navier-Stokes equation (1) can similarly be written in terms of a skew-adjoint operator, although there are some complications in handling the divergence-free constraint [39].

A detailed description of the meaning and importance of the Hamiltonian nature of the nonlinear deterministic dynamics and the Poisson bracket associated with $\boldsymbol{S}$ is given in Refs. [2, 27]. For our purposes, the most important property of Hamiltonian dynamics is that it is incompressible in phase space,

$$
\frac{\partial}{\partial u} \cdot \boldsymbol{S}(u)=\frac{\partial}{\partial u} \cdot \boldsymbol{N}^{\star}(u)=\mathbf{0} .
$$

This implies that the dynamics of the inviscid Burgers equation preserves not just functions (such as the Hamiltonian itself) but also phase-space measures (such as the Gibbs distribution), and thus any probability density that is a function of $H$ only is a candidate equilibrium distribution. The inviscid Burgers equation may also be written in Hamiltonian form using the Hamiltonian $H=\int\left(u^{3} / 6\right) d x$ with $S=-\partial_{x}$ [27, 40]. However, in fluctuating hydrodynamics the choice of the coarse-grained Hamiltonian is dictated by statistical mechanics and the equilibrium Gibbs distribution is maintained via the fluctuation-dissipation balance between the viscous and stochastic terms.

\section{B. Discrete Fluctuating Burgers Equation}

The preceding discussion of how the fluctuating Burgers equation can be written in the form of a generic Langevin equation (3) is formal and merely informs our choice of spatial discretization. The discretized $\boldsymbol{u}=\left\{u_{1}, \ldots, u_{N}\right\}$ can be thought of as a finite-volume representation of the field $u(x, t)$ on a regular grid with spacing $\Delta x$, specifically, $u_{j}$ can be thought of as representing the average value of $u(x, t)$ over the interval (cell) $[j \Delta x,(j+1) \Delta x]$. As we already discussed, this is merely a formal association and the actual physical object is the discrete (coarse-grained) $\boldsymbol{u}(t)$ and not the hypothetical $u(x, t)$. Similarly, the spatially-discretized collection of white noise processes $(\Delta x)^{-1 / 2} \mathcal{W}$ can formally be associated with the space-time white noise $\mathcal{Z}$.

We take the coarse-grained Hamiltonian function to be the natural (local equilibrium [29]) discretization of (5),

$$
H(\boldsymbol{u})=\sum_{j=1}^{N} \frac{\Delta x}{2} u_{j}^{2}
$$

We will construct a spatial discretization that leads to a finite-dimensional generic Langevin equation of the form (3)

$$
\partial_{t} \boldsymbol{u}=\boldsymbol{S} \frac{\partial H}{\partial \boldsymbol{u}}+\frac{\nu}{\Delta x} \boldsymbol{D}_{2} \frac{\partial H}{\partial \boldsymbol{u}}+\left(\frac{2 \nu}{\Delta x}\right)^{1 / 2} \boldsymbol{D}_{1} \mathcal{W}(t)
$$

Here $\mathcal{W}$ is a vector of $N_{w}$ independent white-noise processes (formally, time derivatives of independent Wiener processes), $\boldsymbol{D}_{1}$ is a matrix representing the spatial discretization of the divergence operator, such that $\boldsymbol{D}_{2}=-\boldsymbol{D}_{1} \boldsymbol{D}_{1}^{\star}$ 
is a symmetric negative-semidefinite discretization of the Laplacian operator. This system of SODEs has as an invariant distribution the Gibbs distribution (2) if $\boldsymbol{S}$ is an antisymmetric matrix discretizing (6) that satisfies

$$
\left[\frac{\partial}{\partial \boldsymbol{u}} \cdot \boldsymbol{S}(\boldsymbol{u})\right]_{k}=\sum_{j} \frac{\partial S_{j, k}}{\partial u_{j}}=0 \text { for all } k .
$$

We now construct specific finite-difference operators for $\boldsymbol{D}_{1}$ and $\boldsymbol{S}$.

A particularly simple choice that also generalizes to higher dimensions [41] is to associate fluxes with the half-grid points (faces of the grid in higher dimensions), and to define

$$
\left(\boldsymbol{D}_{1} \mathcal{W}\right)_{j}=\frac{\mathcal{W}_{j+\frac{1}{2}}-\mathcal{W}_{j-\frac{1}{2}}}{\Delta x}, \text { giving }\left(\boldsymbol{D}_{1}^{\star} \boldsymbol{u}\right)_{j+\frac{1}{2}}=-\frac{u_{j+1}-u_{j}}{\Delta x} .
$$

This construction gives the familiar three-point discrete Laplacian $(2 d+1$ points in dimension $d)$,

$$
\left(\boldsymbol{D}_{2} \boldsymbol{u}\right)_{j}=\frac{u_{j-1}-2 u_{j}+u_{j+1}}{\Delta x^{2}},
$$

and is therefore an attractive choice that satisfies the discrete fluctuation-dissipation principle [41]. If periodic boundary conditions are imposed, we set $u_{0}=u_{N}$ and $u_{N+1}=u_{1}$ and $\mathcal{W}_{\frac{1}{2}}=\mathcal{W}_{N+\frac{1}{2}}$ (i.e., $N_{w}=N$ ). For Dirichlet boundary conditions we fix $u_{0}$ and $u_{N+1}$ at specified values and do not need to impose any boundary conditions on $\mathcal{W}$ (i.e., $\left.N_{w}=N+1\right)$.

A natural choice for $\boldsymbol{S}$ is formed by choosing a skew-adjoint discretization $\widetilde{\boldsymbol{D}}_{1}=-\widetilde{\boldsymbol{D}}_{1}^{\star}$ of $\partial_{x}$, in general different from $\boldsymbol{D}_{1}$, and discretizing (6) directly as

$$
(\boldsymbol{S u})_{j}=-\frac{c}{3}\left[u_{j}\left(\widetilde{\boldsymbol{D}}_{1} u\right)_{j}-\left(\widetilde{\boldsymbol{D}}_{1}^{\star} u^{2}\right)_{j}\right]=-\frac{c}{3}\left[u_{j}\left(\widetilde{\boldsymbol{D}}_{1} u\right)_{j}+\left(\widetilde{\boldsymbol{D}}_{1} u^{2}\right)_{j}\right],
$$

where $u^{2}=\left\{u_{1}^{2}, \ldots, u_{N}^{2}\right\}$. We choose $\widetilde{\boldsymbol{D}}_{1}$ to be the second-order centered difference operator

$$
\left(\widetilde{\boldsymbol{D}}_{1} \boldsymbol{u}\right)_{j}=\frac{u_{j+1}-u_{j-1}}{2 \Delta x},
$$

leading to an explicit expression that makes it clear that $\boldsymbol{S u}$ is a discretization of $-c u u_{x}$,

$$
(\boldsymbol{S u})_{j}=-\frac{c}{3}\left[u_{j}\left(\frac{u_{j+1}-u_{j-1}}{2 \Delta x}\right)+\frac{u_{j+1}^{2}-u_{j-1}^{2}}{2 \Delta x}\right]=-c\left(\frac{u_{j-1}+u_{j}+u_{j+1}}{3}\right)\left(\frac{u_{j+1}-u_{j-1}}{2 \Delta x}\right) .
$$

The above discretization of the advective term has been considered frequently in the literature, as discussed in detail in Ref. 40. It can be seen as a weighted combination of the "convective" and the "conservative" forms of advection [42] with weights $1 / 3$ and $2 / 3$, which is the unique choice of weights that gives a conservative and skew-adjoint discretization of advection. It is important to note that one can write the nonlinear term in conservative form,

$$
(\boldsymbol{S u})_{j}=-\frac{c}{2}\left(\frac{u_{j+\frac{1}{2}}^{2}-u_{j-\frac{1}{2}}^{2}}{\Delta x}\right) \text {, where } u_{j+\frac{1}{2}}^{2}=\frac{u_{j}^{2}+u_{j} u_{j+1}+u_{j+1}^{2}}{3} .
$$

Due to the skew-symmetry, in the absence of viscosity the total "energy" 8 is conserved for periodic systems. It can also easily be shown that that the condition (7) is satisfied and therefore this particular discretization of the advective term preserves the Hamiltonian structure of the equations [40].

Putting the pieces together we can write the semi-discrete fluctuating Burgers equation as a system of SODEs, $j=1, \ldots, N$,

$$
\begin{aligned}
\frac{d u_{j}}{d t} & =-\frac{c}{6 \Delta x}\left(u_{j-1}+u_{j}+u_{j+1}\right)\left(u_{j+1}-u_{j-1}\right) \\
& +\frac{\nu}{\Delta x^{2}}\left(u_{j-1}-2 u_{j}+u_{j+1}\right)+\frac{(2 \nu)^{1 / 2}}{\Delta x^{3 / 2}}\left(\mathcal{W}_{j+\frac{1}{2}}(t)-\mathcal{W}_{j-\frac{1}{2}}(t)\right) .
\end{aligned}
$$

With periodic boundary conditions, this stochastic method of lines 24] discretization strictly conserves the total energy (8) and the total momentum

$$
m(\boldsymbol{u})=\sum_{j=1}^{N} \Delta x u_{j}
$$


The equilibrium distribution is the discrete Gibbs-Boltzmann distribution

$$
P_{\text {eq }}(\boldsymbol{u})=Z^{-1} \exp \left[-\frac{\Delta x}{2} \sum_{j=1}^{N} u_{j}^{2}\right] \delta\left(\Delta x \sum_{j=1}^{N} u_{j}-m_{0}\right),
$$

where $m_{0}$ is the initial value for the total momentum. In the next section, we construct efficient temporal discretizations of (13) that preserve these properties as well as possible.

\section{WEAKLY-ACCURATE TEMPORAL INTEGRATORS}

In this section, we consider weak temporal integrators for coupled systems of stochastic ordinary differential equations (SODEs) that arise after spatial discretization of the equations of fluctuating hydrodynamics, such as the system (13). To make the discussion more general and applicable to a large range of generic Langevin equations, we consider the system of nonlinear additive-noise differential equations

$$
\frac{d \boldsymbol{x}}{d t}=\boldsymbol{a}(\boldsymbol{x})+\boldsymbol{K} \mathcal{W}(t)=[\boldsymbol{L}(\boldsymbol{x})] \boldsymbol{x}+\boldsymbol{g}(\boldsymbol{x})+\boldsymbol{K} \mathcal{W}(t) .
$$

There are $N_{v}$ independent variables $\boldsymbol{x}(t)$, and $\mathcal{W}(t)$ denotes a collection of $N_{w}$ independent white-noise processes, formally identified with the time derivative of a collection of independent Brownian motions (Wiener processes). Here $\boldsymbol{K}$ is a constant matrix, and we have used the more natural differential notation since there is no difference between the different stochastic interpretations (e.g., Ito and Stratonovich). For fluctuating hydrodynamics applications, we split the drift $\boldsymbol{a}(\boldsymbol{x})$ into a diffusive term $[\boldsymbol{L}(\boldsymbol{x})] \boldsymbol{x}$ and an advective term $\boldsymbol{g}(\boldsymbol{x})$. This becomes particularly important when considering semi-implicit temporal discretizations since diffusion often needs to be treated implicitly for stability reasons. In general, $\boldsymbol{L}(\boldsymbol{x})$ may depend on $\boldsymbol{x}$ since the transport coefficients (e.g., viscosity) may depend on certain state variables (e.g., density).

Multiplicative noise poses well-known difficulties with constructing higher-order temporal integrators [43. There are many important fluctuating hydrodynamic equations with additive noise, such as the fluctuating Navier-Stokes equation (1), in which $\boldsymbol{L}(\boldsymbol{x}) \equiv \boldsymbol{L}=$ const is a multiple of the (discrete) Laplacian and $\boldsymbol{K}(\boldsymbol{x}) \equiv \boldsymbol{K}=$ const. is a multiple of the (discrete) divergence. For generality in Appendix A we also consider constructing first-order accurate schemes for the case of multiplicative noise, where $\boldsymbol{K}(\boldsymbol{x})$ also depends on $\boldsymbol{x}$, and here we focus on second-order accuracy for additive noise.

There is an extensive literature on numerical methods for finite-dimensional systems of SDEs. At the same time, efficient numerical solution of the types of systems of SDEs appearing in the stochastic method of lines [24] for fluctuating hydrodynamic equations limits the choices of practicable techniques. One of the most important characteristics of systems such as 13 is the presence of a large number of length scales and associated relaxation times (hydrodynamic modes). This intrinsic stiffness is particularly prominent for diffusive terms and makes the construction of stochastic integrators particularly challenging. A powerful class of integrators for systems with a broad spectrum of relevant time scales are exponential integrators [44, 45]. These integrators apply a local linearization [46] and use the matrix exponential $\exp (\boldsymbol{L} \Delta t)$ to capture the dynamics at a time step $\Delta t$ that under-resolves the stiffest modes. Unfortunately, the computation of the matrix exponential is only practicable when it is simple to diagonalize $\boldsymbol{L}$, as is the case when a Fourier basis is used for periodic systems [47. Multi-step schemes are widely used in the deterministic context because of their favorable stability properties and reduced computational effort per time step [48, 49]. Weak multistep schemes have not received much attention in the literature, and their analysis requires developing novel techniques that are outside the scope of this work.

With these considerations in mind, we focus here on one-step Runge-Kutta schemes. They have the advantage of potentially attaining high order of accuracy without requiring evaluation of derivatives of $\boldsymbol{a}(\boldsymbol{x})$. We will consider numerical schemes that only require the generation of $N_{w}=O\left(N_{v}\right)$ Gaussian random variables and do not require the solution of nonlinear systems of equations. While this limits the robustness of the schemes in the nonlinear setting, it is the only type of method that is practicable for the sort of large systems of SODEs that arise when discretizing hydrodynamic SPDEs. First we discuss the simpler case of explicit stochastic Runge-Kutta integrators, and then we consider stochastic variants of two-stage implicit-explicit Runge-Kutta (IMEX-RK) [50] integrators.

\section{A. Conditions for Second-Order Weak Accuracy}

In this section we discuss second-order weakly accurate one-step temporal discretizations with a constant time step $\Delta t$, and denote the numerical approximation $\boldsymbol{x}^{n} \approx \boldsymbol{x}(n \Delta t)$. The most fundamental temporal integrator for (14) is 
the weakly first-order accurate Euler-Maruyama scheme,

$$
\boldsymbol{x}^{n+1}=\boldsymbol{x}^{n}+\Delta t \boldsymbol{a}^{n}+\Delta t^{\frac{1}{2}} \boldsymbol{K}^{n} \boldsymbol{W}^{n}
$$

where the superscript denotes the time level at which the term is evaluated, for example, $\boldsymbol{a}^{n} \equiv \boldsymbol{a}\left(\boldsymbol{x}^{n}\right)$. Here $\boldsymbol{W}^{n}$ is a vector of $m$ independent standard Gaussian variates (i.e., normally-distributed pseudorandom numbers with mean zero and unit variance), generated independently at each time step. The SDE (14) can, in fact, be defined through the limit $\Delta t \rightarrow 0$ of the scheme $\sqrt{15}$ ). The stochastic term $\Delta t^{\frac{1}{2}} \boldsymbol{W}^{n}$ represents the (Wiener) increment of the underlying Brownian motions over the time step. Alternatively, one can view (15) as an application of the deterministic explicit Euler method to (14, with the discrete white noise $\Delta t^{-1 / 2} \boldsymbol{W}^{n}$ representing the rough forcing $\mathcal{W}(t)$. This viewpoint is particularly useful when extending higher-order standard deterministic schemes to the stochastic context.

Let us consider one-step schemes for the general nonlinear additive-noise system of SDEs (14). The general theory of weak accuracy for stochastic integrators is well-established and reviewed, for example, in Section 2.2 of [51]. The key result is that, under certain assumptions, second-order weak accuracy is achieved if the first $2 \cdot 2+1=5$ moments of the numerical increment $\Delta \boldsymbol{x}^{n}=\boldsymbol{x}^{n+1}-\boldsymbol{x}^{n}$ match the moments of the increment $\boldsymbol{x}(n \Delta t+\Delta t)-\boldsymbol{x}(n \Delta t)$ to order $O\left(\Delta t^{2}\right)$. The required moments can be obtained from the well-known weak expansion

$x_{\alpha}(n \Delta t+\Delta t)=x_{\alpha}(n \Delta t)+\Delta t^{\frac{1}{2}} K_{\alpha \beta} W_{\beta}^{n}+\Delta t a_{\alpha}^{n}+\frac{1}{2}\left(\Delta t^{2} a_{\gamma}^{n}+\Delta t^{\frac{3}{2}} K_{\gamma \epsilon} W_{\epsilon}^{n}\right)\left(\partial_{\gamma} a_{\alpha}^{n}\right)+\frac{\Delta t^{2}}{4} K_{\gamma \epsilon} K_{\delta \epsilon}\left(\partial_{\gamma} \partial_{\delta} a_{\alpha}^{n}\right)+O\left(\Delta t^{5 / 2}\right)$,

where a repeated index implies summation and the short-hand notation $\partial_{\gamma} \equiv \partial / \partial x_{\gamma}$ is employed. This expansion is not directly useful for numerical approximations since it requires evaluating derivatives of the drift function. Instead, we employ Runge-Kutta schemes and then ensure second-order weak accuracy by matching the moments of the numerical increments to (16). The details of these calculations are given in Appendix A

A well-known weakly second-order accurate predictor-corrector scheme that is consistent with the conditions derived in Appendix $\mathrm{A}$ is a two-stage explicit trapezoidal method [36, 43, 52. In this scheme the first stage is an EulerMaruyama predictor, and the corrector stage is an explicit trapezoidal rule,

$$
\begin{aligned}
\tilde{\boldsymbol{x}}^{n+1} & =\boldsymbol{x}^{n}+\Delta t \boldsymbol{a}^{n}+\Delta t^{\frac{1}{2}} \boldsymbol{K} \boldsymbol{W}^{n} \\
\boldsymbol{x}^{n+1} & =\boldsymbol{x}^{n}+\frac{\Delta t}{2}\left(\tilde{\boldsymbol{a}}^{n+1}+\boldsymbol{a}^{n}\right)+\Delta t^{\frac{1}{2}} \boldsymbol{K} \boldsymbol{W}^{n}
\end{aligned}
$$

One can naively obtain this scheme from the classical deterministic predictor-corrector algorithm by thinking of $\boldsymbol{F}^{n}=\Delta t^{-1 / 2} \boldsymbol{K} \boldsymbol{W}^{n}$ as a constant applied forcing. The fully explicit nature of this method severely restricts the time step due to stability limits arising from the stiff diffusive terms in fluctuating hydrodynamics. We will consider semi-implicit RK schemes in more detail in Section IIID.

\section{B. Equilibrium Fluctuation Spectrum}

An important property of Langevin-type equations, including those of fluctuating hydrodynamics, is the existence of a non-trivial stationary distribution (invariant measure). It is important for numerical schemes to have an equilibrium distribution that is in, some appropriate sense, close to that of the stochastic differential equations. A recently proposed-approach [53] is to add a Metropolis-Hastings acceptance-rejection rule to a classical integrator such as the Euler-Maruyama scheme. This "Metropolization" ensures that the equilibrium distribution of the numerical approximation is controlled; however, this is done at the cost of reducing the temporal accuracy because of rejections. It is therefore important to ensure that the non-Metropolized numerical scheme produces a good approximation to the equilibrium distribution, so that rejections are infrequent.

Mattingly et al. [54] show that in some appropriate metric the invariant measure (which is assumed to exist) of the numerical scheme has the same order of accuracy as the weak order of accuracy over finite time intervals. This only provides an asymptotic error bound, however, and does not provide an estimate of the actual error. By focusing on the linearized equations of fluctuating hydrodynamics one can easily obtain explicit estimates for the invariant measure of a given numerical scheme and thus understand the nature of discretization errors in the long-time dynamics. This approach was used by some of us in an earlier publication [41] to analyze and improve explicit Runge-Kutta schemes for compressible fluctuating hydrodynamics. Here we briefly review the main results and discuss some generalizations.

We consider the linear system of additive-noise SDEs

$$
\frac{d \boldsymbol{x}}{d t}=\boldsymbol{L} \boldsymbol{x}+\boldsymbol{K} \boldsymbol{W}(t)
$$


A general linear one-step temporal scheme for this equation has the form

$$
\boldsymbol{x}^{n+1}=\boldsymbol{Q} \boldsymbol{x}^{n}+\Delta t^{\frac{1}{2}} \boldsymbol{R} \boldsymbol{W}^{n},
$$

where $\boldsymbol{Q}$ and $\boldsymbol{R}$ are some iteration matrices. Since this is a linear equation forced by a Gaussian process, the solution is a Gaussian process. The equilibrium or steady-state covariance $\boldsymbol{C}_{\Delta t}=\left\langle\boldsymbol{x}^{n}\left(\boldsymbol{x}^{n}\right)^{\star}\right\rangle$ of this linear iteration is the solution of the linear system (see, for example, the derivation in [41])

$$
Q C_{\Delta t} Q^{\star}-C_{\Delta t}=-\Delta t R R^{\star}
$$

In the limit $\Delta t \rightarrow 0$ any consistent and stable numerical scheme should give the correct equilibrium covariance $\boldsymbol{C}=\left\langle\boldsymbol{x}(t) \boldsymbol{x}^{\star}(t)\right\rangle$, which is the solution to [1, 41, 55]

$$
L C+C L^{\star}=-K K^{\star} .
$$

Equation (18) can easily be solved explicitly to obtain an exact exponential integrator for which $\boldsymbol{Q}=\exp (\boldsymbol{L} \Delta t)$ follows from the deterministic variation-of-constants formula. This exponential scheme will be an exact integrator for (18) if and only if

$$
\boldsymbol{R} \boldsymbol{R}^{\star}=\Delta t^{-1}\left[\boldsymbol{C}-\boldsymbol{Q} \boldsymbol{C} \boldsymbol{Q}^{\star}\right]=\Delta t^{-1}\left[\boldsymbol{C}-\exp (\boldsymbol{L} \Delta t) \boldsymbol{C} \exp \left(\boldsymbol{L}^{\star} \Delta t\right)\right] .
$$

In general, one cannot write an explicit solution to this equation unless one can explicitly diagonalize $\boldsymbol{L}$ and $\boldsymbol{C}$ in some basis.

\section{Implicit Midpoint Rule}

Runge-Kutta schemes approximate the matrix exponential $\exp (\boldsymbol{L} \Delta t)$ with a polynomial (for fully explicit schemes) or a rational (for semi-implicit schemes) approximation. An important example is provided by the implicit midpoint (equivalently, trapezoidal) method (Crank-Nicolson scheme) applied to the linear problem (18),

$$
\boldsymbol{x}^{n+1}=\boldsymbol{x}^{n}+\frac{\Delta t}{2} \boldsymbol{L}\left(\boldsymbol{x}^{n}+\boldsymbol{x}^{n+1}\right)+\Delta t^{\frac{1}{2}} \boldsymbol{K} \boldsymbol{W}^{n} .
$$

In this scheme the iteration matrix $\boldsymbol{Q}$ is a $1-1$ Pade approximation of the matrix exponential,

$$
\boldsymbol{Q}=\left(\boldsymbol{I}-\frac{\boldsymbol{L} \Delta t}{2}\right)^{-1}\left(\boldsymbol{I}+\frac{\boldsymbol{L} \Delta t}{2}\right)=\exp (\boldsymbol{L} \Delta t)+O\left(\Delta t^{3}\right)
$$

and $\boldsymbol{R}=(\boldsymbol{I}-\boldsymbol{L} \Delta t / 2)^{-1} \boldsymbol{K}$. It is not hard to show that the implicit midpoint scheme leads to the correct equilibrium covariance $\boldsymbol{C}$ for any time step size since

$$
\boldsymbol{R} \boldsymbol{R}^{\star}=\Delta t^{-1}\left[\boldsymbol{C}-\boldsymbol{Q C} \boldsymbol{Q}^{\star}\right],
$$

as seen from a straightforward explicit calculation,

$$
\begin{aligned}
& \Delta t^{-1}\left[\boldsymbol{C}-\boldsymbol{Q} \boldsymbol{C} \boldsymbol{Q}^{\star}\right] \\
= & \Delta t^{-1}\left(\boldsymbol{I}-\frac{\boldsymbol{L} \Delta t}{2}\right)^{-1}\left[\left(\boldsymbol{I}-\frac{\boldsymbol{L} \Delta t}{2}\right) \boldsymbol{C}\left(\boldsymbol{I}-\frac{\boldsymbol{L}^{\star} \Delta t}{2}\right)-\left(\boldsymbol{I}+\frac{\boldsymbol{L} \Delta t}{2}\right) \boldsymbol{C}\left(\boldsymbol{I}+\frac{\boldsymbol{L}^{\star} \Delta t}{2}\right)\right]\left(\boldsymbol{I}-\frac{\boldsymbol{L}^{\star} \Delta t}{2}\right)^{-1} \\
= & \left(\boldsymbol{I}-\frac{\boldsymbol{L} \Delta t}{2}\right)^{-1}\left(-\boldsymbol{L} \boldsymbol{C}-\boldsymbol{C} \boldsymbol{L}^{\star}\right)\left(\boldsymbol{I}-\frac{\boldsymbol{L}^{\star} \Delta t}{2}\right)^{-1}=\left(\boldsymbol{I}-\frac{\boldsymbol{L} \Delta t}{2}\right)^{-1} \boldsymbol{K} \boldsymbol{K}^{\star}\left(\boldsymbol{I}-\frac{\boldsymbol{L}^{\star} \Delta t}{2}\right)^{-1}=\boldsymbol{R} \boldsymbol{R}^{\star} .
\end{aligned}
$$

An alternative derivation of the fact that 22 gives the correct steady-state covariance for any time step size $\Delta t$ can be found in the Appendix of Ref. 25. That derivation is based on showing that the iteration (22) is a Metropolis-Hastings Monte Carlo algorithm to sample the invariant distribution of (18).

The implicit midpoint rule can easily be generalized to the nonlinear system (14),

$$
\boldsymbol{x}^{n+1}=\boldsymbol{x}^{n}+\Delta t \boldsymbol{a}\left(\frac{\boldsymbol{x}^{n+1}+\boldsymbol{x}^{n}}{2}\right)+\Delta t^{\frac{1}{2}} \boldsymbol{K} \boldsymbol{W}^{n},
$$


which can be shown to be weakly second-order accurate. This scheme is a particularly good candidate for solving Langevin-type equations because it is a time-reversible and quasi-symplectic integrator [23] that exactly conserves all quadratic invariants (e.g., a quadratic Hamiltonian). However, it requires the solution of a nonlinear system of equations at every time step. This nonlinear system of equations may not have a unique solution and is in general too expensive to solve for large-scale hydrodynamic calculations. In the special case of the stochastic Burgers or Navier-Stokes equations, the only nonlinearity in $\boldsymbol{a}(\boldsymbol{x})$ comes from the advective term, which has the special form (to within irrelevant constants) $[\boldsymbol{S}(\boldsymbol{x})] \boldsymbol{x}$ and can be linearized as $\left[\boldsymbol{S}\left(\boldsymbol{x}^{n+\frac{1}{2}}\right)\right] \boldsymbol{x}$, where $\boldsymbol{x}^{n+\frac{1}{2}}$ is a mid-point estimate that has to be obtained via a predictor stage. Such an approach gives a scheme that only requires solving a linear systems in each time step, while still preserving quadratic invariants (e.g., total kinetic energy). It is, however, not a time-reversible scheme. Furthermore, solving the non-symmetric systems that arise when advection is discretized in a semi-implicit manner poses a significant linear algebra challenge, especially when constraints such as incompressibility are included. For this reason, in the next section we consider implicit-explicit Runge-Kutta schemes in which only diffusive terms are, potentially, discretized implicitly.

\section{Fully Explicit Runge-Kutta Scheme}

We now illustrate how the conditions derived in Appendix A can be used in practice to construct a fully-explicit three-stage Runge-Kutta (RK3) integrator. In Ref. [33, an algorithm for the solution of the compressible equations of fluctuating hydrodynamics was proposed, which is based on a well-known three-stage, low-storage total variation diminishing Runge-Kutta (RK3) scheme [56. The RK3 scheme is a simple discretization for the deterministic compressible Navier-Stokes equations that is stable in the inviscid limit, even when slope-limiters are omitted for the convective terms. A stochastic version of the RK3 scheme was analyzed in Ref. 41] based on a linearized analysis, and employed in Ref. [25] along with a staggered spatial discretization. In Ref. [41, nonlinearities were not considered and therefore the scheme presented there is not second-order weakly accurate for nonlinear equations.

Each time step of the RK3 algorithm is composed of three stages, the first one estimating $\boldsymbol{x}$ at time $t=(n+1) \Delta t$, the second at $t=\left(n+\frac{1}{2}\right) \Delta t$, and the final stage obtaining a third-order accurate estimate at $t=(n+1) \Delta t$. Each stage consists of an Euler-Maryama step followed by weighted averaging with the value from the previous stage,

$$
\begin{aligned}
\tilde{\boldsymbol{x}}^{n+1} & =\boldsymbol{x}^{n}+\Delta t \boldsymbol{a}^{n}+\Delta t^{\frac{1}{2}} \boldsymbol{K}\left(\alpha_{1} \boldsymbol{W}_{A}^{n}+\beta_{1} \boldsymbol{W}_{B}^{n}\right) \\
\widetilde{\boldsymbol{x}}^{n+\frac{1}{2}} & =\frac{3}{4} \boldsymbol{x}^{n}+\frac{1}{4}\left[\widetilde{\boldsymbol{x}}^{n+1}+\Delta t \tilde{\boldsymbol{a}}^{n+1}+\Delta t^{\frac{1}{2}} \boldsymbol{K}\left(\alpha_{2} \boldsymbol{W}_{A}^{n}+\beta_{2} \boldsymbol{W}_{B}^{n}\right)\right] \\
\boldsymbol{x}^{n+1} & =\frac{1}{3} \boldsymbol{x}^{n}+\frac{2}{3}\left[\widetilde{\boldsymbol{x}}^{n+\frac{1}{2}}+\Delta t \tilde{\boldsymbol{a}}^{n+\frac{1}{2}}+\Delta t^{\frac{1}{2}} \boldsymbol{K}\left(\alpha_{3} \boldsymbol{W}_{A}^{n}+\beta_{3} \boldsymbol{W}_{B}^{n}\right)\right] .
\end{aligned}
$$

Here $\boldsymbol{W}_{A}^{n}$ and $\boldsymbol{W}_{B}^{n}$ are two independent vectors of i.i.d. normal random variates that are generated independently at each RK3 step, and the weights $\boldsymbol{\alpha}$ and $\boldsymbol{\beta}$ are to be determined. In principle one could use a third sample $\boldsymbol{W}_{C}^{n}$; however, this increases the cost of the method and is insufficient to yield a weakly third-order accurate scheme. Following Ref. [57, we can fix $\alpha_{3}$ and $\beta_{3}$ by making the arbitrary choice that after all the stages are combined the stochastic increment in $\boldsymbol{x}^{n+1}$ be $\Delta t^{\frac{1}{2}} \boldsymbol{K} \boldsymbol{W}_{A}^{n}$.

Runge-Kutta schemes of the above form have been analyzed in Ref. [57] and the moment conditions for weak accuracy of any order derived. Up to second-order one can easily obtain these conditions by explicit Taylor series expansion and comparison of the moments of the numerical increment to those in (16). This gives two quadratic equations for the weights $\boldsymbol{\alpha}$ and $\boldsymbol{\beta}$. Two more equations can be obtained by asking for third-order accuracy of the static covariance $\boldsymbol{C}_{\Delta t}=\boldsymbol{C}+\Delta t^{3} \Delta \boldsymbol{C}+O\left(\Delta t^{4}\right)$ in the linear case. This simple calculation consists of applying the RK3 scheme to the linear equation (18) in order to extract an explicit expression for $\boldsymbol{Q}$ and $\boldsymbol{R}$, and then substituting these expressions in (19) and using (20) to eliminate $\boldsymbol{K} \boldsymbol{K}^{\star}$. By equating the coefficients in front of terms of lower order in $\Delta t$, we can ensure that the error in the stationary covariance is of order $\Delta t^{3}$. In particular, equating the coefficients in front of the terms involving $\boldsymbol{L}^{3} \boldsymbol{C}$ and $\boldsymbol{L}^{2} \boldsymbol{C} \boldsymbol{L}^{\star}$ to zero gives two additional quadratic equations for the weights $\boldsymbol{\alpha}$ and $\boldsymbol{\beta}$. The solution of the resulting system of four equations for the weights $\alpha_{1}, \alpha_{2}, \beta_{1}$ and $\beta_{2}$ gives a 
stochastic RK3 scheme that is of weak order two in the general case and of order three in the linear case,

$$
\begin{aligned}
& \alpha_{1}=\alpha_{2}=\alpha_{3}=1 \\
& \beta_{1}=\frac{(2 \sqrt{2} \pm \sqrt{3})}{5} \\
& \beta_{2}=\frac{(-4 \sqrt{2} \pm 3 \sqrt{3})}{5} \\
& \beta_{3}=\frac{[\sqrt{2} \mp 2 \sqrt{3}]}{10} .
\end{aligned}
$$

For fluctuating hydrodynamics applications, we recommend the upper sign since it gives better discrete static structure factors for a model one-dimensional stochastic advection-diffusion equation (see Ref. 41] for an illustration of this type of calculations).

The RK3 scheme (24) suffers from a severe time step limitation due to the explicit handling of the diffusive terms. We consider alternative semi-implicit methods next.

\section{Semi-Implicit Runge-Kutta Temporal Integrators}

In this section, we construct two-stage second-order implicit-explicit Runge-Kutta schemes for solving a system of SDEs

$$
\frac{d \boldsymbol{x}}{d t}=\boldsymbol{L}(\boldsymbol{x}) \boldsymbol{x}+\boldsymbol{g}(\boldsymbol{x})+\boldsymbol{K} \mathcal{W}(t),
$$

where $\boldsymbol{g}(\boldsymbol{x})$ denotes all of the terms handled explicitly (e.g., advection or external forcing). We will consider schemes that at time step $n$ require solving only linear systems involving the matrix $\boldsymbol{L}^{n}=\boldsymbol{L}\left(\boldsymbol{x}^{n}\right)$, and are parametrized by a vector of weights $\boldsymbol{w}$ (Butcher tableau). The first stage in any of these schemes is a predictor step to estimate $\tilde{\boldsymbol{x}} \approx \boldsymbol{x}\left(n \Delta t+w_{2} \Delta t\right)$, where $w_{2}$ is some chosen weight (e.g., $w_{2}=1 / 2$ for a midpoint predictor). The corrector completes the step by estimating $\boldsymbol{x}^{n+1}$ at time $(n+1) \Delta t$,

$$
\begin{aligned}
\tilde{\boldsymbol{x}} & =\boldsymbol{x}^{n}+\left(w_{2}-w_{1}\right) \Delta t \boldsymbol{L}^{n} \boldsymbol{x}^{n}+w_{1} \Delta t \boldsymbol{L}^{n} \tilde{\boldsymbol{x}}+w_{2} \Delta t \boldsymbol{g}^{n}+\left(w_{2} \Delta t\right)^{\frac{1}{2}} \boldsymbol{K} \boldsymbol{W}_{1}^{n}, \\
\boldsymbol{x}^{n+1} & =\boldsymbol{x}^{n}+\left(1-w_{3}-w_{4}\right) \Delta t \boldsymbol{L}^{n} \boldsymbol{x}^{n}+w_{3} \Delta t \boldsymbol{L}^{n} \tilde{\boldsymbol{x}}+w_{4} \Delta t \boldsymbol{L}^{n} \boldsymbol{x}^{n+1} \\
& +w_{5} \Delta t\left(\widetilde{\boldsymbol{L}}-\boldsymbol{L}^{n}\right) \tilde{\boldsymbol{x}}+w_{5} \Delta t \tilde{\boldsymbol{g}}+\left(1-w_{5}\right) \Delta t \boldsymbol{g}^{n} \\
& +\left(w_{2} \Delta t\right)^{\frac{1}{2}} \boldsymbol{K} \boldsymbol{W}_{1}^{n}+\left(\left(1-w_{2}\right) \Delta t\right)^{\frac{1}{2}} \boldsymbol{K} \boldsymbol{W}_{2}^{n} .
\end{aligned}
$$

The intuition behind the handling of the stochastic increments in the predictor/corrector stages is that $\left(w_{2} \Delta t\right)^{1 / 2} \boldsymbol{W}_{1}^{n}$ samples the increment of the underlying Wiener processes over the time interval $w_{2} \Delta t$, while $\left(\left(1-w_{2}\right) \Delta t\right)^{1 / 2} \boldsymbol{W}_{2}^{n}$ represents the independent increment over the remainder of the time step.

According to the calculations detailed in Appendix A, in order to be second-order weakly accurate in the case of additive noise, the weights $\boldsymbol{w}$ should satisfy the conditions

$$
w_{2} w_{5}=\frac{1}{2}, \quad w_{2} w_{3}+w_{4}=\frac{1}{2} .
$$

We now catalog some possible choices of these weights that satisfy these conditions. We will test the performance of these schemes on the fluctuating Burgers and Navier-Stokes equations in Section $\mathrm{V}$.

\section{Explicit Midpoint Predictor-Corrector Scheme}

If $w_{1}=0$ and $w_{4}=0$ we obtain fully explicit schemes. In this case $w_{3}=w_{5}$ and therefore the splitting into implicit and explicit parts does not matter, and the only function that appears in the scheme is $\boldsymbol{a}(\boldsymbol{x})=\boldsymbol{L}(\boldsymbol{x}) \boldsymbol{x}+\boldsymbol{g}(\boldsymbol{x})$. The only parameter of choice is $w_{2}$. A well-known method that fits the above format is the explicit trapezoidal predictorcorrector method, $w_{2}=1$, and therefore $w_{5}=w_{3}=\frac{1}{2}$. This is exactly the scheme (17). For linear equations, this scheme gives static covariances accurate to second-order, $\boldsymbol{C}_{\Delta t}=\boldsymbol{C}+\Delta t^{2} \Delta \boldsymbol{C}+O\left(\Delta t^{3}\right)$. A notable advantage of this scheme is that it requires generating only a single random increment per time step. 
In order to choose the "best" $w_{2}$, we can look at the accuracy of the static covariance in the linear case, just as we did for the RK3 scheme in Section IIIC. A simple calculation shows that in order to obtain third-order accuracy of the static covariance, $\boldsymbol{C}_{\Delta t}=\boldsymbol{C}+\Delta t^{3} \Delta \boldsymbol{C}+O\left(\Delta t^{4}\right)$, we need to use a mid-point predictor stage, $w_{2}=1 / 2$ and therefore $w_{5}=w_{3}=1$. This gives the explicit midpoint predictor-corrector method

$$
\begin{aligned}
\tilde{\boldsymbol{x}}^{n+\frac{1}{2}} & =\boldsymbol{x}^{n}+\frac{\Delta t}{2} \boldsymbol{a}^{n}+\left(\frac{\Delta t}{2}\right)^{\frac{1}{2}} \boldsymbol{K} \boldsymbol{W}_{1}^{n} \\
\boldsymbol{x}^{n+1} & =\boldsymbol{x}^{n}+\Delta t \tilde{\boldsymbol{a}}^{n+\frac{1}{2}}+\left(\frac{\Delta t}{2}\right)^{\frac{1}{2}} \boldsymbol{K}\left(\boldsymbol{W}_{1}^{n}+\boldsymbol{W}_{2}^{n}\right),
\end{aligned}
$$

which requires generating two random increments per time step. It is important to point out that for advectiondiffusion problems the time step $\Delta t$ needs to be substantially smaller than the stability limit for the explicit midpoint scheme (27) to realize its asymptotic order of accuracy and give substantially more accurate static covariances than the explicit trapezoidal scheme (17) [41].

\section{Implicit Trapezoidal Predictor-Corrector Scheme}

We now extend the implicit midpoint scheme 22 to the case when some terms, such as diffusion, are handled implicitly. In Ref. 25] the fluctuating Navier-Stokes equation was solved using a scheme for which $w_{1}=w_{4}=w_{5}=$ $1 / 2, w_{2}=1, w_{3}=0$, giving the implicit trapezoidal predictor-corrector method,

$$
\begin{aligned}
\tilde{\boldsymbol{x}}^{n+1} & =\boldsymbol{x}^{n}+\frac{\Delta t}{2} \boldsymbol{L}^{n}\left(\boldsymbol{x}^{n}+\tilde{\boldsymbol{x}}^{n+1}\right)+\Delta t \boldsymbol{g}^{n}+\Delta t^{\frac{1}{2}} \boldsymbol{K} \boldsymbol{W}^{n} \\
\boldsymbol{x}^{n+1} & =\boldsymbol{x}^{n}+\frac{\Delta t}{2} \boldsymbol{L}^{n}\left(\boldsymbol{x}^{n}+\boldsymbol{x}^{n+1}\right)+\frac{\Delta t}{2}\left(\widetilde{\boldsymbol{L}}^{n+1}-\boldsymbol{L}^{n}\right) \tilde{\boldsymbol{x}}^{n+1}+\frac{\Delta t}{2}\left(\tilde{\boldsymbol{g}}^{n+1}+\boldsymbol{g}^{n}\right)+\Delta t^{\frac{1}{2}} \boldsymbol{K} \boldsymbol{W}^{n} .
\end{aligned}
$$

If $\boldsymbol{g}=\mathbf{0}$ this scheme is stable for any time step, more precisely, it is $A$-stable (see Appendix B for $L$-stable schemes). The scheme has the great advantage that for the linearized equation 18 it gives the correct stationary covariance regardless of the time step, while only requiring a single random increment per time step. We emphasize that the dynamics of the fluctuations is not correctly reproduced for large time step sizes, as we discuss further in Appendix B.

\section{Implicit Midpoint Predictor-Corrector Scheme}

In the scheme (28), a trapezoidal approximation is used for the explicit fluxes, just as in (17). Another candidate is to use a midpoint approximation for the explicit fluxes, as in (27), obtained by using $w_{2}=1 / 2$ and therefore $w_{5}=1$. We also choose a corrector stage where the implicit part is the implicit midpoint rule 22, which requires choosing $w_{3}=0$ and $w_{4}=1 / 2$. We are left with a choice for $w_{1}$ in the predictor stage,

$$
\begin{aligned}
\tilde{\boldsymbol{x}}^{n+\frac{1}{2}} & =\boldsymbol{x}^{n}+\left(\frac{1}{2}-w_{1}\right) \boldsymbol{L}^{n} \boldsymbol{x}^{n} \Delta t+w_{1} \boldsymbol{L}^{n} \tilde{\boldsymbol{x}}^{n+\frac{1}{2}}+\frac{\Delta t}{2} \boldsymbol{g}^{n}+\left(\frac{\Delta t}{2}\right)^{\frac{1}{2}} \boldsymbol{K} \boldsymbol{W}_{1}^{n} \\
\boldsymbol{x}^{n+1} & =\boldsymbol{x}^{n}+\frac{\Delta t}{2} \boldsymbol{L}^{n}\left(\boldsymbol{x}^{n}+\boldsymbol{x}^{n+1}\right)+\Delta t\left(\widetilde{\boldsymbol{L}}^{n+\frac{1}{2}}-\boldsymbol{L}^{n}\right) \tilde{\boldsymbol{x}}^{n+\frac{1}{2}}+\Delta t \tilde{\boldsymbol{g}}^{n+\frac{1}{2}}+\left(\frac{\Delta t}{2}\right)^{\frac{1}{2}} \boldsymbol{K}\left(\boldsymbol{W}_{1}^{n}+\boldsymbol{W}_{2}^{n}\right) .
\end{aligned}
$$

Two obvious choices are $w_{1}=1 / 2$, which for linear equations makes the predictor stage a backward Euler step with time step size $\Delta t / 2$. An alternative is to use $w_{1}=1 / 4$, which for linear equations makes the predictor stage an implicit midpoint step with time step size $\Delta t / 2$. For the linearized equation (18) the predictor stage does not actually matter since it is only used in evaluating the nonlinear terms in the corrector stage. Therefore, we will compare the implicit midpoint schemes with $w_{1}=1 / 4$ and $w_{1}=1 / 2$ numerically in Section $\mathrm{V}$.

\section{FLUCTUATING NAVIER-STOKES EQUATION}

The implicit-explicit schemes discussed in Section IIID are general schemes suitable for unconstrained SDEs and cannot directly be applied to the fluctuating Navier-Stokes equation (1). Some care is required in handling the 
incompressibility constraint in a computationally-efficient manner without compromising the stochastic accuracy. The spatio-temporal discretization we analyze here was proposed and applied in Ref. [25]; here we provide additional analysis and a discussion of alternative approaches.

\section{A. Continuum Equations}

In principle, the incompressibility constraint can be most easily handled by using a projection operator formalism to eliminate pressure from (1] and write the fluctuating Navier-Stokes equation in the form

$$
\partial_{t} \boldsymbol{v}=\mathcal{P}\left[-\boldsymbol{v} \cdot \boldsymbol{\nabla} \boldsymbol{v}+\nu \boldsymbol{\nabla}^{2} \boldsymbol{v}+\left(2 \nu \rho^{-1} k_{B} T\right)^{\frac{1}{2}} \boldsymbol{\nabla} \cdot \mathcal{Z}_{\boldsymbol{v}}\right]
$$

Here $\mathcal{P}$ is the orthogonal projection onto the space of divergence-free velocity fields, $\mathcal{P}=\boldsymbol{I}-\mathcal{G}(\mathcal{D} \mathcal{G})^{-1} \mathcal{D}$ in real space, where $\mathcal{D} \equiv \boldsymbol{\nabla}$. denotes the divergence operator and $\mathcal{G} \equiv \boldsymbol{\nabla}$ the gradient operator with the appropriate boundary conditions taken into account. We only consider periodic, no-slip and free-slip boundaries. With periodic boundaries we can express all operators in Fourier space and $\widehat{\mathcal{P}}=\boldsymbol{I}-k^{-2}\left(\boldsymbol{k} \boldsymbol{k}^{\star}\right)$, where $\boldsymbol{k}$ is the wavenumber. The application of the projection to the right hand side ensures that $\boldsymbol{\nabla} \cdot \boldsymbol{v}=0$ at all times if the initial condition is divergence free. The divergence-free constraint is a constant linear constraint and the projection restricts the velocity dynamics to the constant linear subspace of divergence-free vector fields. The projection operator can be applied in more general settings, notably, in cases where the constraints are nonlinear and the noise is multiplicative; however, the resulting expressions are rather complex especially in the stochastic setting [58, 59].

In practice, the fluctuating velocities modeled by 30 advect other quantities, and it is this coupling between the velocity and other equations that is of most interest. Perhaps the simplest example is provided by a stochastic advection-diffusion for the concentration or density $c(\boldsymbol{r}, t)$ of a large collection of non-interacting passive tracers. For example, $c(\boldsymbol{r}, t)$ might corresponds to the light intensity pattern of fluorescently-labeled molecules suspended in the fluid in a Fluorescence Recovery After Photobleaching (FRAP) experiment. In general, the equation for the concentration has multiplicative noise [25]. This arises because the coarse-grained free energy functional $H[\boldsymbol{x}(\boldsymbol{r}, t)]=$ $H(\boldsymbol{v}, c)$ includes a contribution from the entropy of the passive tracer which is, in general, a non-quadratic even if local functional of $c$. For illustration purposes we can take a separable quadratic Hamiltonian (i.e., independent Gaussian fluctuations in velocity and concentration),

$$
H(\boldsymbol{v}, c)=H_{\boldsymbol{v}}(\boldsymbol{v})+H_{c}(c)=\frac{\rho}{2} \int v^{2} d \boldsymbol{r}+\frac{k_{B} T}{2 \epsilon} \int c^{2} d \boldsymbol{r},
$$

and write the the model additive-noise tracer equation

$$
\partial_{t} c=-\boldsymbol{v} \cdot \nabla c+\chi \nabla^{2} c+\nabla \cdot\left[(2 \epsilon \chi)^{\frac{1}{2}} \mathcal{Z}_{c}\right]
$$

The multiplicative noise case is not considered herein. Physically, $\epsilon$ measures the degree of coarse graining, $\epsilon \sim N_{p}^{-1}$, where $N_{p}$ is the number of tracer particles per coarse degree of freedom. Note that (31) is a conservation law because $\boldsymbol{v} \cdot \boldsymbol{\nabla} c=\boldsymbol{\nabla} \cdot(c \boldsymbol{v})$ due to incompressibility.

The coupled velocity-concentration system (30 31) can formally be written in the form (3). The chemical potential $\mu(c)=\partial H / \partial c \sim c$. The mobility operator can be written as a sum of a skew-adjoint and a self-adjoint part,

$$
\boldsymbol{N}=\boldsymbol{M}-\boldsymbol{S}=-\left[\begin{array}{cc}
\rho^{-1} \nu\left(\mathcal{P} \boldsymbol{\nabla}^{2} \mathcal{P}\right) & \mathbf{0} \\
\mathbf{0} & \epsilon\left(k_{B} T\right)^{-1}\left(\chi \boldsymbol{\nabla}^{2}\right)
\end{array}\right]-\rho^{-1}\left[\begin{array}{cc}
(\mathcal{P} \boldsymbol{\omega} \mathcal{P}) & \mathcal{P} \boldsymbol{\nabla} c \\
-(\boldsymbol{\nabla} c)^{T} \mathcal{P} & 0
\end{array}\right]
$$

where $\boldsymbol{\omega}$ is the antisymmetric vorticity tensor, $\boldsymbol{\omega}_{j k}=\partial v_{k} / \partial r_{j}-\partial v_{j} / \partial r_{k}$, and we used the vector identity

$$
\boldsymbol{\omega} \boldsymbol{v}=-(\boldsymbol{\nabla} \times \boldsymbol{v}) \times \boldsymbol{v}=-\boldsymbol{v} \cdot \boldsymbol{\nabla} \boldsymbol{v}+\boldsymbol{\nabla}\left(\frac{v^{2}}{2}\right) .
$$

Even though by skew symmetry the top right sub-block of $\boldsymbol{S}$ is nonzero, there is no coupling of concentration back in the velocity equation because

$$
\left(\frac{\partial H}{\partial c}\right) \nabla c=\left(\frac{d H_{c}}{d c}\right) \nabla c=\nabla H_{c}
$$

is a gradient of a scalar and is eliminated by the projection. The velocity equation therefore remains of the form 30 . 


\section{B. Spatial Discretization}

For a detailed description of the spatial discretization of (30 31) that we employ we refer the reader to Ref. 25]. The discretization of the velocity equation is based on a staggered or MAC grid [60] in which the component of velocity along a given dimension is discretized on a uniform grid that is shifted by half a grid spacing along that dimension. Following the stochastic methods of lines that we used for the Burgers equation in Section IIB the spatial discretization of (1) leads to a system of SODEs of the form

$$
\frac{d \boldsymbol{v}}{d t}=\mathbb{P}\left[\boldsymbol{S}_{\boldsymbol{v}}(\boldsymbol{v}) \boldsymbol{v}+\nu \boldsymbol{L}_{\boldsymbol{v}} \boldsymbol{v}+\left(\frac{2 \nu k_{B} T}{\rho \Delta V}\right)^{\frac{1}{2}} \boldsymbol{D}_{\boldsymbol{w}} \mathcal{W}_{\boldsymbol{v}}(t)\right]
$$

where $\boldsymbol{S}_{\boldsymbol{v}}(\boldsymbol{v})$ denotes a discretization of the advective operator $-(\boldsymbol{v} \cdot \boldsymbol{\nabla}), \Delta V$ is the volume of a hydrodynamic cell, and $\mathcal{W}_{\boldsymbol{v}}(t)$ is a collection of white-noise processes [25]. Here $\boldsymbol{D}_{\boldsymbol{w}}$ a tensor divergence operator that applies the conservative discrete vector divergence operator $\boldsymbol{D}$ independently for each coordinate, $\boldsymbol{L}_{\boldsymbol{v}}$ is a discrete (vector) Laplacian, and $\mathbb{P}=\boldsymbol{I}-\boldsymbol{G}(\boldsymbol{D} \boldsymbol{G})^{-1} \boldsymbol{D}$ is a discrete projection operator, where $\boldsymbol{G}$ is a discrete scalar gradient operator. The imposed periodic, no-slip or free-slip boundary conditions are encoded in the specific forms of the discrete difference operators near the boundaries of the domain.

Let us first focus on creeping Stokes flow, where the advective term $\boldsymbol{v} \cdot \boldsymbol{\nabla} \boldsymbol{v}$ is neglected. Following the same procedure as we employed for the fluctuating Burgers equation in Section II B, the spatial discretization is constructed to obey a discrete fluctuation-dissipation balance principle. This relies on several key properties of the staggered difference operators. Importantly, the discrete gradient and divergence operators obey the duality relation $\boldsymbol{G}=-\boldsymbol{D}^{\star}$, just as the continuum operators. The resulting scalar Laplacian $\boldsymbol{L}_{s}=\boldsymbol{D} \boldsymbol{G}=-\boldsymbol{D} \boldsymbol{D}^{\star}$ is the standard $(2 d+1)$-point discrete Laplacian, which is also applied to each (staggered) component of the velocity to form $\boldsymbol{L}_{\boldsymbol{v}}=-\boldsymbol{D}_{\boldsymbol{w}}\left(\boldsymbol{D}_{\boldsymbol{w}}\right)^{\star}(\operatorname{see}$ Ref. 25] for a discussion of modifications near physical boundaries). Because of the duality between $\boldsymbol{D}$ and $\boldsymbol{G}$ the MAC projection is self-adjoint, $\mathbb{P}^{\star}=\mathbb{P}$, and idempotent, $\mathbb{P}^{2}=\mathbb{P}$, just like the continuum projection operator. From these properties and Eq. 200 it follows (see Appendix in Ref. [25] for details) that the equilibrium covariance of the fluctuating velocities is

$$
\left\langle\boldsymbol{v} \boldsymbol{v}^{\star}\right\rangle=\frac{k_{B} T}{\rho \Delta V} \mathbb{P}
$$

This means that when an equilibrium snapshot of the velocity is expressed in any orthonormal basis for the subspace of discretely divergence-free vector fields, the coefficients are i.i.d. Gaussian random variables with mean zero and variance $\rho^{-1} k_{B} T / \Delta V$. This is the expression of discrete fluctuation-dissipation balance for the case of incompressible flow.

The addition of the nonlinear advective term does not affect the discrete fluctuation-dissipation balance since the advective term is skew-adjoint, $\boldsymbol{S}_{\boldsymbol{v}}^{\star}=-\boldsymbol{S}_{\boldsymbol{v}}$, just like the discretization 12 of the term $u u_{x}$ for the one-dimensional case. Specifically, in two dimensions, for a given $\boldsymbol{u}$ such that $\boldsymbol{D} \boldsymbol{u}=0$, the spatial discretization of the advective term described in Refs. [25, 42, leads to

$$
\begin{aligned}
{\left[\boldsymbol{S}_{\boldsymbol{v}}(\boldsymbol{u}) \boldsymbol{v}\right]_{i+\frac{1}{2}, j}^{(x)}=} & -(4 \Delta x)^{-1}\left[\left(u_{i+\frac{3}{2}, j}^{(x)}+u_{i+\frac{1}{2}, j}^{(x)}\right) v_{i+\frac{3}{2}, j}^{(x)}-\left(u_{i-\frac{1}{2}, j}^{(x)}+u_{i+\frac{1}{2}, j}^{(x)}\right) v_{i-\frac{1}{2}, j}^{(x)}\right] \\
& -(4 \Delta y)^{-1}\left[\left(u_{i, j+\frac{1}{2}}^{(y)}+u_{i+1, j+\frac{1}{2}}^{(y)}\right) v_{i+\frac{1}{2}, j+1}^{(x)}-\left(u_{i, j-\frac{1}{2}}^{(y)}+u_{i+1, j-\frac{1}{2}}^{(y)}\right) v_{i+\frac{1}{2}, j-1}^{(x)}\right]
\end{aligned}
$$

This can easily be shown to be a skew-adjoint discretization, $\left[\boldsymbol{S}_{\boldsymbol{v}}(\boldsymbol{u}) \boldsymbol{v}\right] \cdot \boldsymbol{w}=-\left[\boldsymbol{S}_{\boldsymbol{v}}(\boldsymbol{u}) \boldsymbol{w}\right] \cdot \boldsymbol{v}$, for either periodic, free-slip and no-slip conditions (or any combination thereof). Furthermore, this discretization leads to Hamiltonian dynamics for inviscid flow, i.e., the phase-space flow generated by the advective term is incompressible,

$$
\frac{\partial}{\partial \boldsymbol{v}} \cdot \boldsymbol{S}_{\boldsymbol{v}}(\boldsymbol{v})=\mathbf{0}
$$

A good temporal integrator should preserve this special structure of the equations and reproduce the correct velocity fluctuations for reasonably large time step sizes.

Note that the addition of a passively-advected scalar field poses no additional difficulties if $c$ is discretized on the regular (non-staggered) grid underlying the (staggered) velocity grid. Specifically, the spatial discretization of (31) that we employ is a scalar equivalent of 33 ,

$$
\frac{d \boldsymbol{c}}{d t}=\boldsymbol{S}_{c}(\boldsymbol{v}) \boldsymbol{c}+\chi \boldsymbol{L}_{c} \boldsymbol{c}+\left(\frac{2 \epsilon \chi}{\Delta V}\right)^{\frac{1}{2}} \boldsymbol{D} \mathcal{W}_{c}(t)
$$


where $\boldsymbol{S}_{c}(\boldsymbol{v})$ is a cell-centered conservative and skew-adjoint discretization of the advection operator $-(\boldsymbol{v} \cdot \boldsymbol{\nabla})$,

$$
\left[\boldsymbol{S}_{c}(\boldsymbol{u}) \boldsymbol{c}\right]_{i, j}=-(2 \Delta x)^{-1}\left(u_{i+\frac{1}{2}, j}^{(x)} c_{i+1, j}-u_{i-\frac{1}{2}, j}^{(x)} c_{i-1, j}\right)-(2 \Delta y)^{-1}\left(u_{i, j+\frac{1}{2}}^{(y)} c_{i, j+1}-u_{i, j-\frac{1}{2}}^{(y)} c_{i, j-1}\right),
$$

and $\boldsymbol{L}_{c}$ is equivalent to $\boldsymbol{L}_{s}=\boldsymbol{D} \boldsymbol{G}$ except near boundaries. The semi-discrete equation (35) obeys a discrete fluctuationdissipation balance principle 25. Specifically, at thermodynamic equilibrium the fluctuations in the concentration are Gaussian with covariance

$$
\left\langle\boldsymbol{c c ^ { \star }}\right\rangle=\frac{\epsilon}{\Delta V} \boldsymbol{I},
$$

and also uncorrelated with the velocity fluctuations.

The coupled velocity-concentration equation system of SODEs $(3335)$ can be written in the generic Langevin form (3). Note that the concentration-dependent term in the velocity equation that ought to be included to preserve the skew-symmetry of the non-diffusive terms can be written in the form of a projected discrete gradient of a scalar, $\mathbb{P} \boldsymbol{G}\left(c^{2} / 2\right)$, which vanishes identically. This shows that in two dimensions the system 33 35) is time reversible with respect to the equilibrium Gibbs-Boltzmann distribution with the separable discrete Hamiltonian

$$
H(\boldsymbol{v}, c)=\frac{\rho \Delta V}{2} \sum_{i, j}\left[\left(v_{i+\frac{1}{2}, j}^{(x)}\right)^{2}+\left(v_{i, j+\frac{1}{2}}^{(y)}\right)^{2}\right]+\frac{k_{B} T \Delta V}{2 \epsilon} \sum_{i, j} c_{i, j}^{2} .
$$

Note that the Gibbs-Boltzmann distribution is constrained to the linear subspace of discretely divergence-free vector fields,

$$
(\boldsymbol{D} \boldsymbol{v})_{i, j}=\Delta x^{-1}\left(v_{i+\frac{1}{2}, j}^{(x)}-v_{i-\frac{1}{2}, j}^{(x)}\right)+\Delta y^{-1}\left(v_{i, j+\frac{1}{2}}^{(y)}-v_{i, j-\frac{1}{2}}^{(y)}\right)=0,
$$

and with periodic boundaries both the average momentum and average concentration are conserved by the dynamics.

\section{Temporal Discretization}

In our initial discussion of temporal integration schemes for $(33)$ we will neglect the advective term and focus on creeping Stokes flow, thus avoiding technical details while preserving the essential features of the problem. There is a vast literature on deterministic temporal integration of the incompressible Navier-Stokes equations, and, in particular, the handling of the $\nabla \pi$ term. One of the most popular class of methods are splitting or projection methods, such as the prototype projected Euler-Maruyama method,

$$
\boldsymbol{v}^{n+1}=\boldsymbol{v}^{n}+\nu \Delta t \mathbb{P} \boldsymbol{L}_{\boldsymbol{v}} \boldsymbol{v}^{n}+(2 \nu \Delta t)^{\frac{1}{2}} \mathbb{P} \boldsymbol{D}_{\boldsymbol{w}} \boldsymbol{W}_{\boldsymbol{v}}^{n},
$$

where we set $\rho^{-1} k_{B} T / \Delta V=1$ for simplicity and $\boldsymbol{W}_{\boldsymbol{v}}^{n}$ are i.i.d. standard normal random variates generated independently at each time step. Note that in practice, due to roundoff errors and the use of inexact Poisson solvers in the projection operation, it is preferable to apply $\mathbb{P}$ to $\boldsymbol{v}^{n}$ as well.

As explained in Appendix C (see also Appendix B in Ref. [25]), the iteration (37) gives a steady-state covariance that is a first-order accurate approximation to the continuum result (34),

$$
\boldsymbol{C}_{\boldsymbol{v}}=\left\langle\boldsymbol{v}^{n}\left(\boldsymbol{v}^{n}\right)^{\star}\right\rangle=\mathbb{P}+\Delta t \Delta \boldsymbol{C}_{\boldsymbol{v}}+O\left(\Delta t^{2}\right) .
$$

In Appendix C we consider approximate projection methods [61,62] and find that they do not satisfy this requirement. The scheme (37) can be seen as a direct application of the Euler-Maruyama method to (33). Note that any purely explicit scheme, including the RK3 scheme described in Section III C, can be applied to (33) by simply performing a projection operation after every stage of the scheme.

Semi-implicit schemes can also be applied to (33). As a prototype example, let us consider the implicit midpoint method (22),

$$
\boldsymbol{v}^{n+1}=\boldsymbol{v}^{n}+\frac{\nu \Delta t}{2} \mathbb{P} \boldsymbol{L}_{\boldsymbol{v}}\left(\boldsymbol{v}^{n}+\boldsymbol{v}^{n+1}\right)+(2 \nu \Delta t)^{\frac{1}{2}} \mathbb{P} \boldsymbol{D}_{\boldsymbol{w}} \boldsymbol{W}_{\boldsymbol{v}}^{n} .
$$

At first sight, it appears that solving (38) requires the application of $\left[\boldsymbol{I}-(\nu \Delta t / 2) \mathbb{P} \boldsymbol{L}_{\boldsymbol{v}}\right]^{-1}$. However, it is not hard to see that solving 38 is equivalent to solving the following linear system for the velocity $\boldsymbol{v}^{n+1}$ and the pressure $\boldsymbol{\pi}^{n+\frac{1}{2}}$,

$$
\begin{aligned}
\left(\boldsymbol{I}-\frac{\nu \Delta t}{2} \boldsymbol{L}_{\boldsymbol{v}}\right) \boldsymbol{v}^{n+1}+\Delta t \boldsymbol{G} \boldsymbol{\pi}^{n+\frac{1}{2}} & =\left(\boldsymbol{I}+\frac{\nu \Delta t}{2} \boldsymbol{L}_{\boldsymbol{v}}\right) \boldsymbol{v}^{n}+(2 \nu \Delta t)^{\frac{1}{2}} \boldsymbol{D}_{\boldsymbol{w}} \boldsymbol{W}_{\boldsymbol{v}}^{n} \\
\boldsymbol{D} \boldsymbol{v}^{n+1} & =0 .
\end{aligned}
$$


This coupled velocity-pressure Stokes linear system can be solved efficiently even in the presence of non-periodic boundaries by using a preconditioned Krylov iterative solver, as described in detail in Ref. [63. The scheme (38) reproduces the static covariance of the velocity fluctuations exactly, $\boldsymbol{C}_{\boldsymbol{v}}=\mathbb{P}$, for any time step. A more intuitive approach to analysing the scheme (39) based on computing the modes of the spatial discretization is described in Appendix D.

Having illustrated how the implicit midpoint rule 22 can be applied to the time-dependent Stokes equations, it is simple to modify the implicit-explicit predictor-corrector schemes described in Section IIID to account for incompressibility. In Ref. 25] the implicit trapezoidal predictor-corrector scheme (28) was used to solve (30[31), but only tested in an essentially linearized context. Here we also consider the implicit midpoint predictor-corrector scheme 29 , for which the predictor stage consists of solving the linear system for $\tilde{\boldsymbol{v}}^{n+\frac{1}{2}}, \tilde{\boldsymbol{\pi}}^{n+\frac{1}{2}}$ and $\tilde{\boldsymbol{c}}^{n+\frac{1}{2}}$,

$$
\begin{aligned}
\left(\boldsymbol{I}-w_{1} \nu \Delta t \boldsymbol{L}_{\boldsymbol{v}}\right) \tilde{\boldsymbol{v}}^{n+\frac{1}{2}}+\Delta t \boldsymbol{G} \tilde{\boldsymbol{\pi}}^{n+\frac{1}{2}} & =\frac{\Delta t}{2} \boldsymbol{S}_{\boldsymbol{v}}\left(\boldsymbol{v}^{n}\right) \boldsymbol{v}^{n}+\left[\boldsymbol{I}+\left(\frac{1}{2}-w_{1}\right) \nu \Delta t \boldsymbol{L}_{\boldsymbol{v}}\right] \boldsymbol{v}^{n}+(\nu \Delta t)^{\frac{1}{2}} \boldsymbol{D}_{\boldsymbol{w}}\left(\boldsymbol{W}_{\boldsymbol{v}}^{n}\right)_{1} \\
\left(\boldsymbol{I}-w_{1} \chi \Delta t \boldsymbol{L}_{c}\right) \tilde{\boldsymbol{c}}^{n+\frac{1}{2}} & =\frac{\Delta t}{2} \boldsymbol{S}_{c}\left(\boldsymbol{v}^{n}\right) \boldsymbol{c}^{n}+\left[\boldsymbol{I}+\left(\frac{1}{2}-w_{1}\right) \chi \Delta t \boldsymbol{L}_{c}\right] \boldsymbol{c}^{n}+(\epsilon \chi \Delta t)^{\frac{1}{2}} \boldsymbol{D}\left(\boldsymbol{W}_{c}^{n}\right)_{1} \\
\boldsymbol{D} \tilde{\boldsymbol{v}}^{n+\frac{1}{2}} & =0
\end{aligned}
$$

and similarly for the corrector stage. The concentration equation is decoupled from the velocity equation and can be solved using standard techniques, e.g., multigrid methods. Note that in practice it is better to rewrite the linear systems in terms of the increments $\tilde{\boldsymbol{v}}^{n+\frac{1}{2}}-\boldsymbol{v}^{n}$ and $\tilde{\boldsymbol{c}}^{n+\frac{1}{2}}-\boldsymbol{c}^{n}$. This is because for weak fluctuations the terms involving the identity matrix may dominate the right-hand side and compromise the accuracy of the linear solvers, unless special care is taken in choosing the termination criteria for the iterative linear solvers.

\section{SIMULATIONS}

In this section we numerically study the behavior of the implicit-explicit trapezoidal (28) and midpoint (29) schemes on the fluctuating Burgers and Navier-Stokes equations. We focus here on the behavior of the equilibrium distribution of the fluctuating fields for large fluctuations and large time step sizes. The discrete spectrum of the equilibrium fluctuations is one of the most important properties of a numerical scheme for long-time simulations.

Our spatial discretizations were constructed to obey a discrete fluctuation-dissipation balance principle, which means that for sufficiently small time steps the numerical schemes will produce the correct equilibrium fluctuations even when the nonlinear terms are important. In the absence of advection, the schemes (28) and (29) reduce to the implicit midpoint rule, which was designed to produce the correct equilibrium fluctuations for any time step. It is not a priori obvious how increasing the time step size affects the long-time behavior of schemes in the presence of the nonlinear advective terms, which are treated explicitly. We study this question numerically in this section.

In fluctuating hydrodynamics, the magnitude of the equilibrium fluctuations is controlled by the degree of coarse graining, more specifically, by the average number of particles $N_{p}$ (microscopic degrees of freedom) per hydrodynamic cell (macroscopic degree of freedom). In particular, the law of large numbers suggests that the Gaussian fluctuations at equilibrium have a variance inversely proportional to $N_{p} \sim \Delta V$. In order to model the effect of the degree of coarse graining we can introduce a parameter $\epsilon \sim N_{p}^{-1}$ that measures the strength of the fluctuations, with $\epsilon \sim 1$ indicating very strong fluctuations, i.e., minimal coarse graining. While we cannot expect Markovian SPDE models to be a good approximation to reality in the absence of coarse-graining, from a numerical analysis perspective it is important to understand how robust the numerical schemes are to increased magnitude of the fluctuations. We note that the types of schemes we use here may have uses in other fields such as turbulence, where reproducing the correct spectrum of fluctuations is also important.

\section{A. Fluctuating Burgers Equation}

We first turn our attention to the fluctuating Burgers equation. For simplicity, we take $c=1$ and consider a periodic system with zero total momentum (no macroscopic advection),

$$
\partial_{t} u+u \partial_{x} u=\nu \partial_{x x}^{2} u+(2 \epsilon \nu)^{\frac{1}{2}} \partial_{x} \mathcal{Z} .
$$

When the spatial discretization 13 is used, the coarse-grained velocities $u_{j}$ have Gaussian equilibrium fluctuations with mean zero and covariance

$$
\left\langle u_{i} u_{j}\right\rangle=\frac{\epsilon}{\Delta x} \delta_{i j}
$$


The equilibrium magnitude of the velocities is therefore $|u| \approx \sqrt{\epsilon / \Delta x}$, which is a measure of the typical magnitude of the advection speed.

\section{Dimensionless Numbers}

In deterministic fluid dynamics, the dimensionless number that describes how well advection is resolved by the time step size is the advective CFL (Courant-Friedrichs-Levy) number

$$
\alpha=\frac{|u| \Delta t}{\Delta x} \approx \Delta t \epsilon^{\frac{1}{2}} \Delta x^{-\frac{3}{2}} .
$$

Traditional wisdom says that for schemes that handle advection explicitly $\Delta t$ should be chosen such that $\alpha \lesssim 1$. The presence of diffusion, however, stabilizes numerical schemes by adding dissipation and introduces the viscous CFL number $\beta$ and the cell Reynolds number $r$,

$$
\beta=\frac{\nu \Delta t}{\Delta x^{2}}, \quad r=\frac{\alpha}{\beta}=\frac{|u| \Delta x}{\nu} \approx \epsilon^{\frac{1}{2}} \nu^{-1} \Delta x^{\frac{1}{2}} .
$$

Note that the cell Reynolds number measures the relative importance of the nonlinear term (advection) versus the linear term (diffusion), and is independent of $\Delta t$. In the deterministic setting, standard von Neumann stability analysis for the Euler scheme applied to the advection-diffusion equation suggests that our centered discretization of advection is stable if $\alpha^{2} / 2 \leq \beta \leq 1 / 2$. This suggests that the dimensionless number

$$
\gamma=\frac{\alpha^{2}}{\beta}=r^{2} \beta=\frac{|u|^{2} \Delta t}{\nu} \approx \frac{\epsilon \Delta t}{\nu \Delta x}
$$

may be important in controlling the behavior of our spatio-temporal discretizations.

For weak fluctuations $(\epsilon \ll 1)$ or strong dissipation $(r \ll 1)$, both of which are typically true for realistic fluids at small scales and sufficient levels of coarse-graining $\left(N_{p} \gg 1\right)$, the accuracy is controlled by the viscous CFL number $\beta$. In particular, if $\beta \ll 1$ we can be confident that the numerical scheme resolves the dynamics of the fluctuations accurately. However, running with small viscous CFL numbers is often impractical. Our semi-implicit schemes are designed to be stable and also to correctly reproduce the equilibrium fluctuations even for large time steps, $\beta \gg 1$, at least as long as $\alpha \ll 1$. An important question, which is difficult to analyze with existing analytical tools, is how large the time step can be before the explicit handling of the nonlinear advective terms introduces large errors. We study this question here by examining the equilibrium fluctuations for strong fluctuations, $\epsilon \gtrsim 1$, and large time steps, $\beta \gg 1$.

\section{Static Structure Factors}

In order to study the behavior of the equilibrium fluctuations we follow the approach used in Ref. [41. Instead of studying the fluctuations in the actual (real space) variables $u_{j}$, we study the equilibrium discrete Fourier spectrum of the fluctuating variables, defined as

$$
S_{\kappa}=N \epsilon^{-1} \Delta x\left\langle\hat{u}_{k} \hat{u}_{k}^{\star}\right\rangle .
$$

Here the discrete Fourier transform is defined as

$$
\hat{u}_{k}=N^{-1} \sum_{j=0}^{N} u_{j+1} e^{-i j \Delta k},
$$

where $0 \leq \kappa \leq\lfloor N / 2\rfloor$ is the waveindex and $\Delta k=2 \pi \kappa / N \leq \pi$ is the dimensionless wavenumber. The quantity $S_{\kappa}$ is a dimensionless discrete version of what is called the static structure factor $S(k)$ in the physics literature, where $k=\Delta k / \Delta x$ is the physical wavenumber. For fluctuating hydrodynamics, at thermodynamic equilibrium

$$
S_{\kappa}=1 \text { for all } \kappa \neq 0,
$$

which is a re-statement of the discrete fluctuation-dissipation balance principle. 

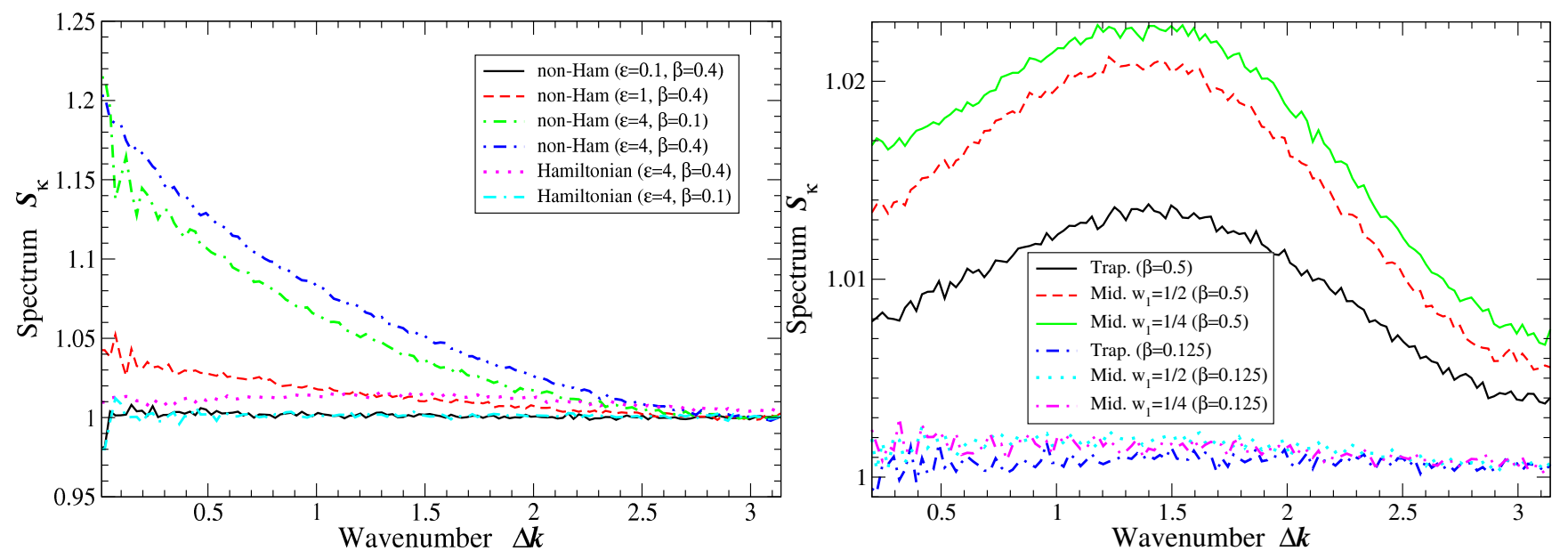

Figure 1: Static structure factor $S_{\kappa}$ for a periodic system of 256 cells at thermodynamic equilibrium. (Left panel) Midpoint scheme (29) with $w_{1}=1 / 2$ and non-Hamiltonian advection for time step size $\beta=0.4$ for weak fluctuations $\epsilon=0.1$ $(\alpha \approx 0.13)$, medium fluctuations $\epsilon=1(\alpha=0.4)$ and strong fluctuations $\epsilon=4(\alpha=0.8)$. For $\epsilon=4$, reducing the time step to $\beta=0.1(\alpha=0.2)$ does not significantly improve the accuracy and even if $\Delta t \rightarrow 0$ the wrong spectrum of fluctuations is obtained. Switching to the Hamiltonian discretization $\sqrt{12}$ significantly lowers the errors. (Right panel) Comparison between the trapezoidal scheme (28) and the midpoint scheme $(29)$ with $w_{1}=1 / 2$ and with $w_{1}=1 / 4$, for $\epsilon=4$ and large time step size $\beta=0.5(\alpha=1)$ and small time step $\beta=0.125(\alpha=0.25)$. Advection is discretized in a Hamiltonian manner.

For periodic systems, due to translational invariance, the quantity $S_{\kappa}$ contains the same statistics about the equilibrium fluctuations as the $N \times N$ covariance matrix $C_{j, j^{\prime}}=\left\langle u_{j} u_{j^{\prime}}\right\rangle$. The advantage of using the Fourier description is that it illustrates the behavior at different physical length scales. It is expected that any numerical scheme will produce some artifacts at the largest wavenumbers because of the strong corrections due to the discretization; however, small wavenumbers, $\Delta k \ll 1$, ought to have much smaller errors because they evolve over time scales and length scales much larger than the discretization step sizes $\Delta x$ and $\Delta t$. A scheme or choice of time step size that produces a discrete structure factor $S_{\kappa}$ much different from unity at small wavenumbers must be rejected as unphysical. It is important to emphasize, however, that getting a good equilibrium static spectrum for the fluctuations is not a guarantee that a scheme accurately models the dynamics of the fluctuations.

\section{Numerical Results}

In Fig. 1 we show numerical results for the equilibrium structure factor $S_{\kappa}$ for a periodic system with $\nu=1$ and $\Delta x=1$ and zero total momentum, $\left\langle u_{j}\right\rangle=0$. To illustrate the importance of using a Hamiltonian discretization of advection in the nonlinear setting, we consider a scheme where the advective term $u u_{x}$ is handled using the conservative but non-Hamiltonian discretization

$$
(\boldsymbol{S u})_{j}=-\frac{c}{2}\left(\frac{u_{j+\frac{1}{2}}^{2}-u_{j-\frac{1}{2}}^{2}}{\Delta x}\right)=-\frac{c}{2}\left(\frac{u_{j+1}^{2}-u_{j-1}^{2}}{2 \Delta x}\right), \text { where } u_{j+\frac{1}{2}}^{2}=\frac{u_{j}^{2}+u_{j+1}^{2}}{2},
$$

instead of the conservative Hamiltonian discretization $(12)$. We recall that the correct answer is $S_{\kappa}=1$ for all wavenumbers. The results in the left panel of Fig. 1 illustrate that for weak fluctuations (i.e., nearly linear equations), the correct spectrum is obtained. However, for strong fluctuations (i.e., nonlinear equations), $\epsilon=4$, the non-Hamiltonian scheme produces the wrong static spectrum of fluctuations at small wavenumbers, and reducing $\Delta t$ does not help.

On the other hand, the Hamiltonian discretization gives small errors in the spectrum even for the larger time step size. In the right panel of Fig. 1 we zoom in to show the magnitude and form of the errors in the structure factors for the implicit-explicit trapezoidal scheme (28) and for the midpoint scheme (29) with $w_{1}=1 / 2$ and with $w_{1}=1 / 4$. We see that all three schemes show similarly small errors in the spectrum. Reducing $\Delta t$ by a factor of four makes the error statistically insignificant. We have verified that the errors are of second order in the time step size $\Delta t$ for all three schemes.

In Fig. 2 we show numerical results for the static structure factor in the case of weaker fluctuations, $\epsilon=0.1$ and $\epsilon=0.01$, but large time step size. This is a typical scenario for fluctuating hydrodynamics in practice, since for 

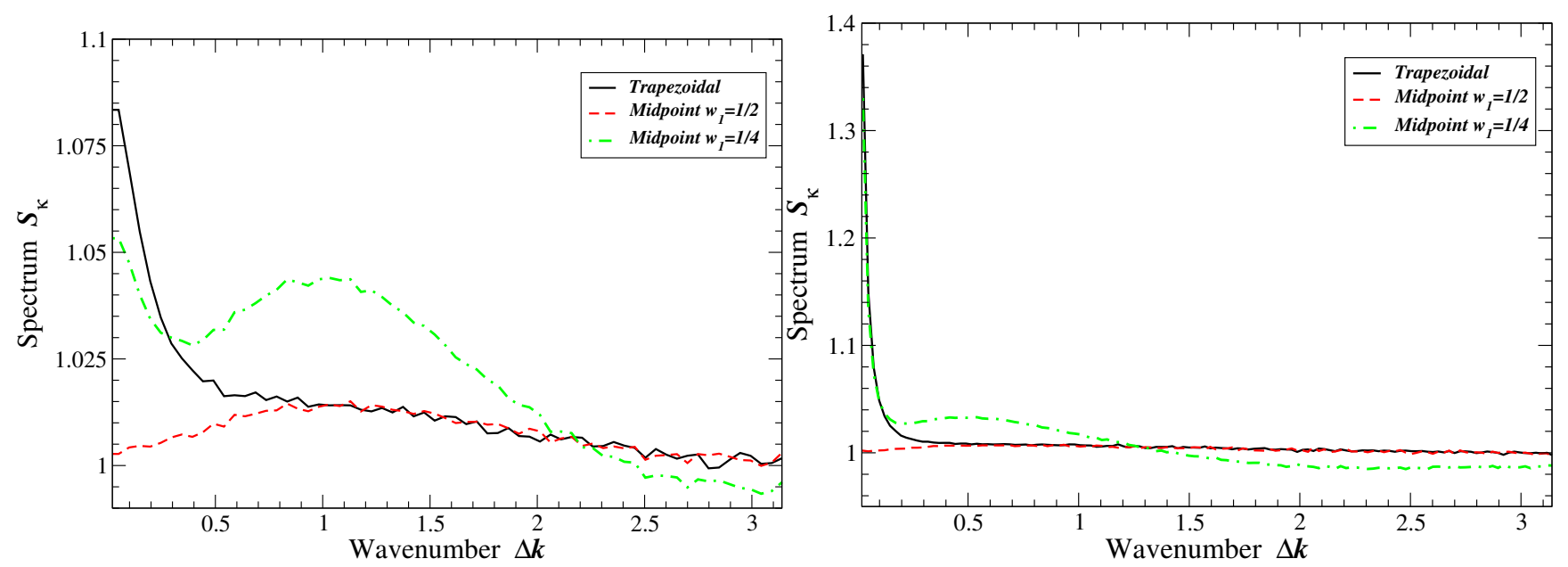

Figure 2: Static structure factor $S_{\kappa}$ at thermodynamic equilibrium in the case of weaker fluctuations and large time step sizes, for the trapezoidal scheme (28) and for the midpoint scheme $\sqrt{29}$ with $w_{1}=1 / 2$ and with $w_{1}=1 / 4$. (Left panel) Moderate fluctuations, $\epsilon=0.1$, for time step size $\beta=10, \alpha \approx 3.2, \gamma=1$. (Right panel) Weak fluctuations, $\epsilon=0.01$ and time time step size, $\beta=100, \alpha=10, \gamma=1$.

reasonable degree of coarse graining the fluctuations would be small and the behavior of the equations would be close to that of the linearized equations. In the absence of the advective nonlinearity our schemes are stable for arbitrary viscous CFL number $\beta$. Under equilibrium conditions the semi-implicit predictor-corrector schemes we consider are observed to be stable up to rather large $\Delta t$ as measured in the advective CFL $\alpha$, and even the dimensionless number $\gamma=\alpha^{2} / \beta$. However, for sufficiently large $\Delta t$ the nonlinearities are expected to play some role, and one cannot expect to be able to increase the time step size up to the stability limit and still maintain reasonable accuracy. The results in Fig. 2 show that for large $\Delta t$ there appear significant artifacts in the static structure factor for the trapezoidal scheme (28) and for the midpoint scheme (29) with $w_{1}=1 / 4$. While the magnitude of the errors is small, the problematic observation is that the errors have a peak at the smallest wavenumbers, where we expect schemes to most closely mimic the continuum equations.

The above observations lead us to select the midpoint scheme $(29)$ with $w_{1}=1 / 2$ as the most robust temporal integrator for the fluctuating Burgers equation. At the same time, we should recognize that the best choice of scheme will depend on the quantity of interest and the particular problem under consideration. All schemes are observed to produce equilibrium fluctuations that are rather robust under the presence of strong non-linearities if a Hamiltonian advection of discretization is employed. In the next section we confirm that this conclusion also holds for the fluctuating Navier-Stokes equation.

\section{B. Fluctuating Navier-Stokes Equation with Passive Tracer}

We now turn our attention to the fluctuating Navier-Stokes equations with a passively-advected scalar, Eqs. (30]31). Here we set $\rho^{-1} k_{B} T=\epsilon$ so that $\epsilon$ controls the magnitude of both the velocity and the concentration fluctuations.

The implementation of our spatio-temporal discretizations and their accuracy in the linearized setting (weak fluctuations) is discussed in more detail in Ref. [25. Our implementation is integrated into the the IBAMR software framework 64, an open-source library for developing fluid-structure interaction models that use the immersed boundary method. Based on our experience with the fluctuating Burgers equation, we focus our attention on the implicit-explicit trapezoidal scheme (28) and the midpoint scheme $(29)$ with $w_{1}=1 / 2$. For simplicity, here we focus on two spatial dimensions and periodic boundary conditions, but we wish to emphasize that our formulation, numerical schemes, and implementation apply to three spatial dimensions and no-slip or free-slip boundaries as well.

For simplicity, in our numerical tests we set $\Delta x=\Delta y=1$. The diffusion coefficients for momentum and concentration are set to $\nu=1$ and $\chi=0.25$, and a grid of size $64 \times 64$ is employed. The same dimensionless numbers as for the Burgers equation apply, with the difference that there is a separate diffusive CFL for the concentration, $\beta_{c}=\chi \beta / \nu$, and therefore the cell Peclet number $r_{c}=|u| \Delta x / \chi$ is four times larger than the cell Reynolds number $r$. 


\section{Static Structure Factors}

The equilibrium fluctuations in velocity and concentration are characterized by the static structure factors, which are the equilibrium average of the discrete Fourier spectrum of the fluctuating velocities and concentrations. Concentration fluctuations are characterized via

$$
S_{\kappa}^{(c)}=N_{c} \epsilon^{-1} \Delta V\left\langle\hat{c}_{\kappa} \hat{c}_{\kappa}^{\star}\right\rangle,
$$

where $\Delta V=\Delta x \Delta y$ is the volume of the hydrodynamic cells, $N_{c}$ is the number of hydrodynamic cells, and $\kappa=\left(\kappa_{x}, \kappa_{y}\right)$ is the waveindex. For the velocity fluctuations, we calculate the spectrum of the fluctuations of a variable related to vorticity [25],

$$
S_{\kappa}^{(\Omega)}=N_{c} \epsilon^{-1} \Delta V\left\langle\hat{\Omega}_{\kappa} \hat{\Omega}_{\kappa}^{\star}\right\rangle,
$$

where $\hat{\Omega}_{\kappa}$ is obtained from the discrete Fourier spectrum of the velocity components as

$$
\hat{\Omega}_{\kappa}=k^{-1}\left(k_{x} \hat{v}_{y}-k_{y} \hat{v}_{x}\right),
$$

and $k=\sqrt{k_{x}^{2}+k_{y}^{2}}$ is the wavenumber. Note that $S_{\kappa}^{(\Omega)}$ fully characterizes the covariance of the velocity fluctuations since our scheme ensures the velocity is discretely divergence free at all times and $k^{-1}\left(-k_{y}, k_{x}\right)$ spans the subspace of divergence-free velocities in Fourier space. For staggered variables the shift between the corresponding grids should be taken into account as a phase shift in Fourier space, for example, exp $\left(k_{x} \Delta x / 2\right)$ for $v_{x}$. Additionally, the wavenumber $\boldsymbol{k}=\left(k_{x}, k_{y}\right)$ should be replaced by the effective wavenumber $\tilde{\boldsymbol{k}}$ that takes into account the centered discretization of the projection operator, for example,

$$
\tilde{k}_{x}=\frac{\exp \left(i k_{x} \Delta x / 2\right)-\exp \left(-i k_{x} \Delta x / 2\right)}{i \Delta x}=k_{x} \frac{\sin \left(k_{x} \Delta x / 2\right)}{\left(k_{x} \Delta x / 2\right)} .
$$

One can additionally define and measure the cross-correlation between concentration and velocity fluctuations via the cross-correlation static structure factor

$$
S_{\kappa}^{(c, \Omega)}=N_{c} \epsilon^{-1} \Delta V\left\langle\hat{c}_{\kappa} \hat{\Omega}_{\kappa}^{\star}\right\rangle,
$$

which is in general a complex number.

Discrete fluctuation-dissipation balance in the coupled velocity-concentration equations requires that $S_{\kappa}^{(c)}=S_{\kappa}^{(\Omega)}=$ 1 and $S_{\boldsymbol{\kappa}}^{(c, v)}=0$ for all nonzero wavenumbers. Deviations from these values indicate a violation of discrete fluctuationdissipation balance and can be used to numerically assess the behavior of the schemes in the nonlinear setting, as we do next.

\section{Numerical Results}

In Fig. 3 we show numerical results for the spectrum of the fluctuations in the solenoidal modes of velocity and concentration for strong fluctuations, $\epsilon=4$. We see that, just as for the fluctuating Burgers equation, both the trapezoidal and the midpoint scheme show artifacts in the spectra, especially for concentration and for the trapezoidal scheme. The cross-correlation $S_{\kappa}^{(c, \Omega)}$ is found to be small and difficult to measure due to large statistical errors.

We have verified that as the time step is reduced, both schemes give the correct spectrum even for strong fluctuations (i.e., strong nonlinearities). In Fig. 4 we show the average error in the equilibrium spectrum $\left\langle\left|S_{\kappa}-1\right|\right\rangle$ for vorticity and concentration. The second-order weak accuracy of the error is clearly seen in Fig. 2 for both velocity and concentration and for both the midpoint and the trapezoidal scheme.

In Fig. 5 we show the spectrum of concentration fluctuations $S_{\kappa}^{(c)}$ for the case of weak fluctuations, $\epsilon=0.01$, and large time step size, viscous CFL $\beta=50$ and diffusive CFL $\beta_{c}=12.5$, and advective CFL $\alpha=5$. We see a large error for small wavenumbers for the trapezoidal scheme. A similar but weaker artifact is seen for the velocity spectrum $S_{\kappa}^{(\Omega)}$ as well, indicating that the trapezoidal scheme violates fluctuation-dissipation balance at small wavenumbers for large $\Delta t$. The midpoint scheme is seen to be much more accurate for both $S_{\kappa}^{(c)}$ and $S_{\kappa}^{(\Omega)}$. These investigations confirm that the midpoint scheme 29 with $w_{1}=1 / 2$ is the more robust temporal integrator for fluctuating hydrodynamics. 

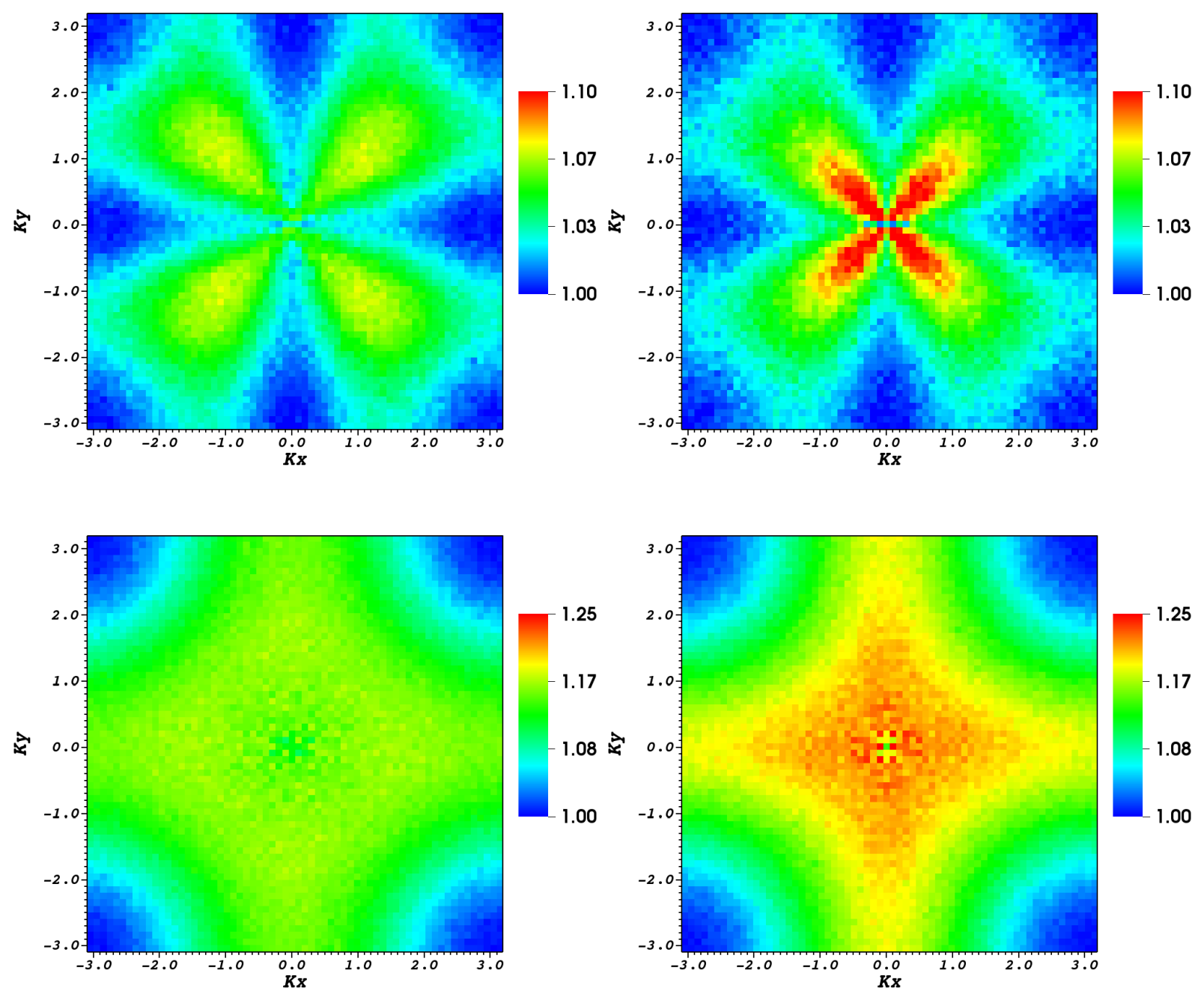

Figure 3: Discrete structure factors $S_{\kappa}^{(\Omega)}$ (top) and $S_{\kappa}^{(c)}$ (bottom) for the midpoint scheme $\left[29\right.$ with $w_{1}=1 / 2$ on the left and for the trapezoidal scheme 28 ) on the right, for the case of large fluctuations $\epsilon=4$. (Top row) Velocity spectra for a time step size $\beta=0.5, \alpha=1$. (Bottom row) concentration spectra for $\beta_{c}=\chi \beta / \nu=0.0625$ and $\alpha=0.5$.

\section{CONCLUSIONS}

Discrete and continuum Langevin models are often used as coarse-grained models for the behavior of materials at mesoscopic scales. These models include the effects of thermal fluctuations via white-noise stochastic forcing terms chosen in a way that ensures fluctuation-dissipation balance. This means that at thermodynamic equilibrium the dynamics is time reversible (i.e., in detailed balance) with respect to the Gibbs-Boltzmann distribution. For continuum models this is usually only true formally and a more precise interpretation of the equations requires introducing a spatial discretization and truncating the continuum models at a scale well-separated from the molecular scale. Here we focused on fluctuating hydrodynamics, specifically, we considered numerical methods for solving the fluctuating Burgers equation in one dimension and the fluctuating Navier-Stokes equations in two dimensions. In these equations, fluctuation-dissipation balance is obtained from the balance of the dissipative (self-adjoint) diffusive terms and the stochastic forcing. The advection terms are non-dissipative (skew-adjoint) and do not affect the equilibrium distribution.

Spatio-temporal discretizations do not necessarily preserve the properties of the continuum equations, notably, they may not obey a discrete fluctuation-dissipation principle. This means that their equilibrium distribution is not a discrete form of the Gibbs-Boltzmann distribution. This sort of unphysical behavior can be avoided by carefully constructing the spatial discretization to obey a discrete-fluctuation principle with respect to a target discrete Gibbs- 

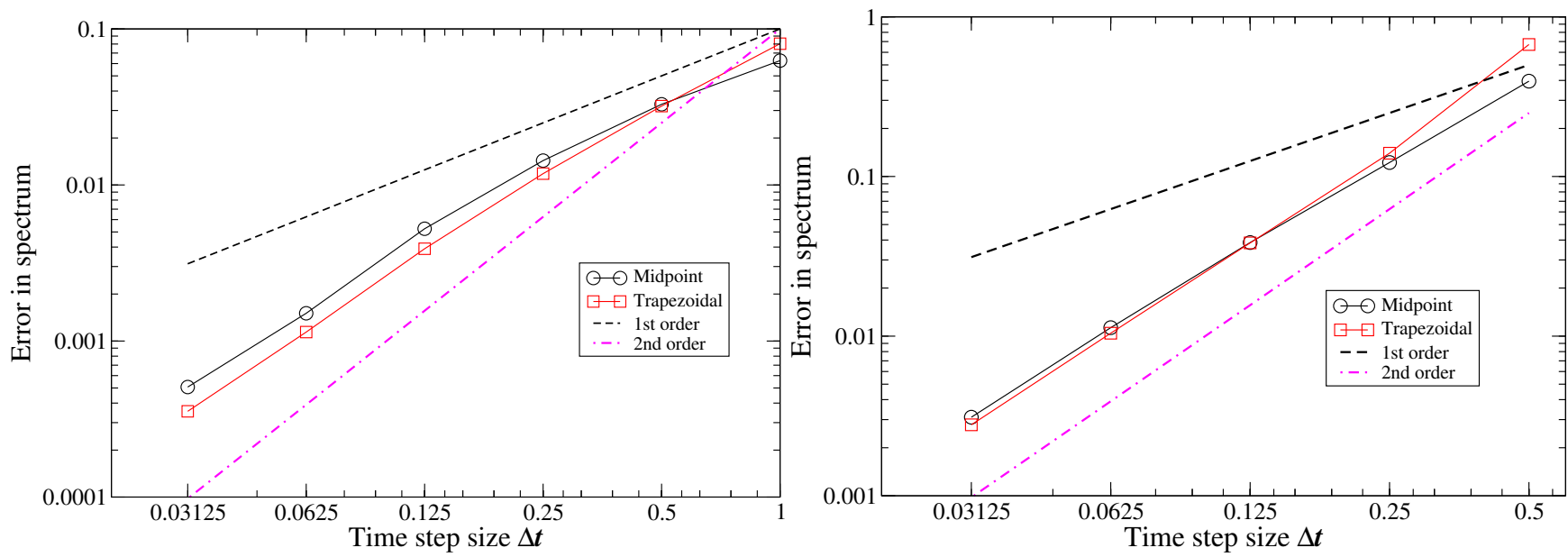

Figure 4: Average error in the static spectra for several time step sizes for the trapezoidal scheme $(28)$ and for the midpoint scheme 29 with $w_{1}=1 / 2$, for the case of large fluctuations $\epsilon=4$. First- and second-order error trends are indicated, showing the second-order asymptotic accuracy. Error bars are comparable to the symbol size and not shown. (Left panel) $\left\langle S_{\kappa}^{(\Omega)}-1\right\rangle$. (Right panel) $\left\langle S_{\kappa}^{(c)}-1\right\rangle$.
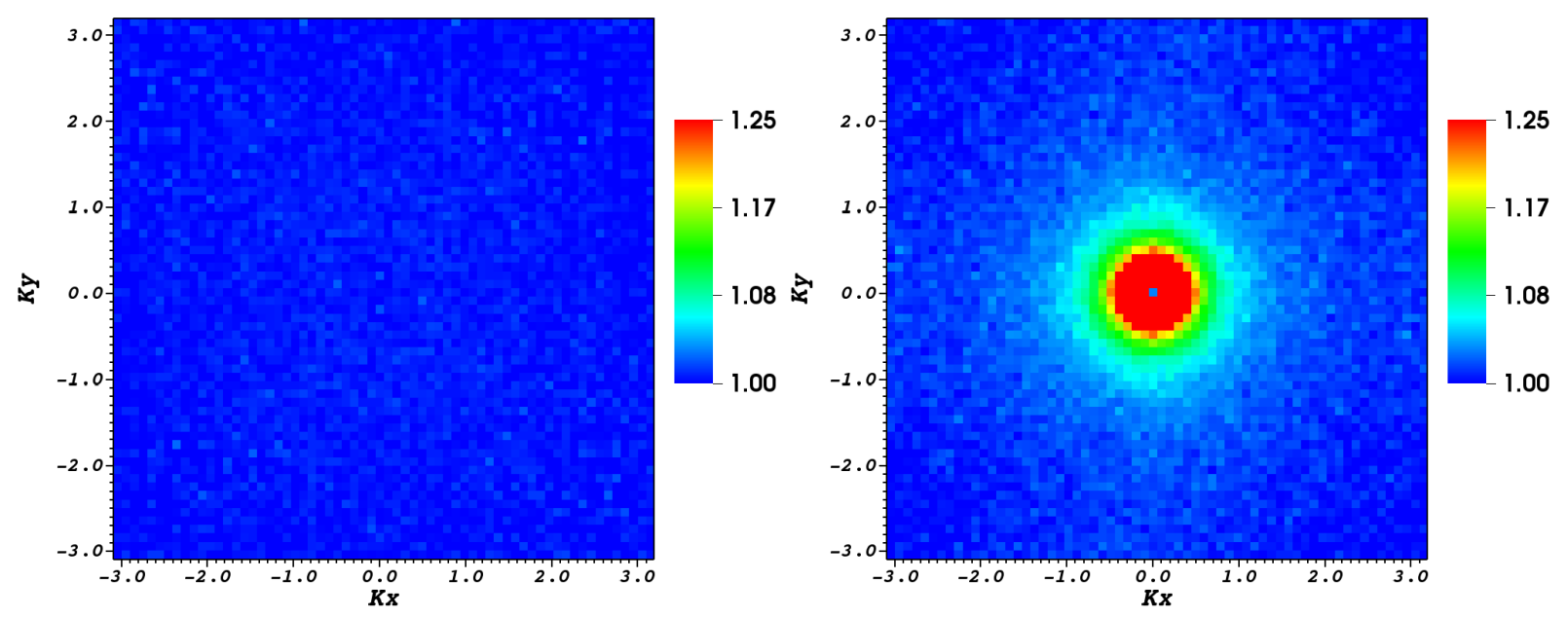

Figure 5: Discrete structure factor for concentration $S_{\kappa}^{(c)}$ for the midpoint scheme 29 with $w_{1}=1 / 2$ on the left and for the trapezoidal scheme (28) on the right, for the case of weak fluctuations, $\epsilon=0.01$, and large time step size $\beta=50, \beta_{c}=12.5$, $\alpha=5$.

Boltzmann distribution. In this way, a coarse-grained semi-discrete model is obtained that obeys the principles of statistical mechanics, namely, obeys detailed balance at thermodynamic equilibrium. We showed explicitly how one can construct such spatial discretizations by first understanding the properties of the continuum model (even if only at a formal level) and then maintaining those properties in the spatial discretization. For fluctuating hydrodynamics, this means that certain relations between the discrete divergence, gradient and Laplacian operators must be preserved, such as the fact that the divergence and gradient operators are negative adjoints of each other. We showed how to construct spatial discretizations that obey a discrete fluctuation-dissipation principle for both the fluctuating Burgers and the fluctuating Navier-Stokes with the addition of a passively-advected scalar. These results were mostly a summary of discretizations previously constructed in somewhat disjoint bodies of literature, and are a stochastic equivalent of a method-of-lines approach for deterministic fluid dynamics [24].

The novel challenge that we tackled here is the construction of temporal integrators that are weakly second-order accurate for additive noise, and preserve the fluctuation-dissipation balance reasonably accurately. It is possible to construct temporal integrators that strictly preserve the Gibbs-Boltzmann distribution at equilibrium by combining 
a Metropolis-Hastings rejection procedure with a more classical temporal integrator. However, effective use of such Metropolization [53] requires first constructing a classical weak integrator that produces a good approximation to the equilibrium distribution for time step sizes that are only limited by the deterministic dynamics. For separable Langevin equations, it is well-known how to construct symplectic integrators that preserve dynamical invariants, including the equilibrium distribution, very robustly [23]. One of the simplest second-order methods of this type suitable for fluctuating hydrodynamics is the implicit midpoint rule. However, an implicit discretization of the nonlinear advective terms is arguably impractical in three dimensions. Many existing deterministic schemes in fluid dynamics use a mixed implicit-explicit approach in which the diffusive terms are handled implicitly and the advective terms are handled explicitly. We showed how to construct predictor-corrector second-order Runge-Kutta schemes based on the implicit midpoint rule (Crank-Nicolson method) for the diffusive terms, and either an explicit midpoint or an explicit trapezoidal rule for the advective terms. These schemes have the remarkable property that in the linearized setting (weak fluctuations) they exactly preserve the Gaussian approximation to the Gibbs-Boltzmann distribution. We obtained the conditions for second-order weak accuracy of a rather general class of two-stage RungeKutta methods, and proposed several specific schemes. Through numerical investigations, we found the scheme (29), which combines the implicit and explicit midpoint rules, was very robust in reproducing the correct spectrum of fluctuations, even for strong fluctuations (strong nonlinearities) and for large time step sizes.

A remaining challenge is how to control not just the static distribution of the equilibrium fluctuations but also to control the accuracy of the dynamics of the fluctuating fields. This is particularly challenging for the case in which there are several physical processes with large separation of time scales, such as, for example, the diffusion of momentum and of mass. Notably, for many realistic fluids the Schmidt number $S_{c}=\nu / \chi$ is on the order of $10^{2}-10^{3}$, making the sort of integrators we discussed impractical because the time step would be severely limited by the viscosity $\nu$ and not by the diffusion coefficient $\chi$. Temporal integrators for this sort of multiscale, or more appropriately, manyscale system of SPDEs, will be the subject of future investigations. Future work should also explore Metropolization [53] strategies for predictor-corrector schemes for fluctuating hydrodynamics, particularly for the case of multiplicative noise.

We focused here on constructing temporal integrators with weak accuracy of order two for additive equations. This choice is guided in part by physical intuition that has yet to be justified more rigorously. Specifically, fluctuating hydrodynamics is typically applied at length and time scales where fluctuations are weak and the dynamics is in a nearly linearized regime. Notably, in many cases of interest the noise is essentially additive or can be well-approximated as additive. Weak accuracy is emphasized because it is more relevant in the types of Monte Carlo applications we are interested in. While the classical definition of strong order of accuracy is likely too strong for Monte Carlo simulation, some notion of pathwise convergence is important in reproducing the statistics of typical paths, such as, for example, rare transition statistics. Recently, error metrics other than weak and strong error have also been considered. Notably, Ref. 65] defines the numerical error as the difference between the joint PDF of the computed values at all time steps and the joint PDF of the exact process at the same times, and constructs Runge-Kutta integrators accurate in that metric. Such approaches may be fruitfully applied in fluctuating hydrodynamics in the future, especially in the context of modeling rare events.

\section{Acknowledgments}

We would like to thank John Bell, Alejandro Garcia, and Jonathan Goodman for numerous enlightening discussions. A. Donev was supported in part by the National Science Foundation (NSF) under grant DMS-1115341 and the Office of Science of the U.S. Department of Energy (DOE) Early Career award number de-sc0008271. B. Griffith and S. Delong acknowledge research support from the NSF under award OCI 1047734. E. Vanden-Eijnden and S. Delong were supported by the DOE office of Advanced Scientific Computing Research under grant DE-FG02-88ER25053. Additional support for E. Vanden-Eijnden was provided by the NSF under grant DMS07-08140 and by the Office of Naval Research under grant N00014-11-1-0345.

\section{Appendix A: Weak Semi-Implicit Predictor-Corrector Schemes}

We consider implicit-explicit stochastic Runge-Kutta schemes to solve the system of Ito SODEs

$$
d \boldsymbol{x}=\boldsymbol{L}(\boldsymbol{x}) \boldsymbol{x} d t+\boldsymbol{g}(\boldsymbol{x}) d t+\alpha \partial_{\boldsymbol{x}} \cdot\left[\boldsymbol{K}(\boldsymbol{x}) \boldsymbol{K}^{\star}(\boldsymbol{x})\right] d t+\boldsymbol{K}(\boldsymbol{x}) d \boldsymbol{B}(t),
$$

where $\boldsymbol{L}(\boldsymbol{x})$ is a linear operator that represents the implicitly-treated part of the dynamics. Here $\mathcal{B}$ is a collection of independent Brownian motions (Wiener processes), with $\mathcal{W} \equiv d \mathcal{B} / d t$ denoting a collection of white noise processes. 
For increased generality we allow for multiplicative noise and include a divergence term proportional to some constant $\alpha$, as may be required to ensure fluctuation-dissipation balance in the case of multiplicative noise. For example, $\alpha=1 / 2$ gives the spurious or thermal drift term in the generic Langevin equation (3) if the non-dissipative part of the dynamics is Hamiltonian. We develop a method that is weakly second order accurate for additive noise and first order accurate for multiplicative noise, requires solving only linear systems involving the matrix $\boldsymbol{L}^{n}=\boldsymbol{L}\left(\boldsymbol{x}^{n}\right)$, and does not require evaluating the divergence of the mobility matrix $\boldsymbol{M}=\boldsymbol{K} \boldsymbol{K}^{\star}$.

We employ a two-stage (predictor-corrector) Runge-Kutta method, where at time step $n$ the predictor estimates a first-order accurate solution at an intermediate time $n \Delta t+w_{2} \Delta t$,

$$
\tilde{\boldsymbol{x}}=\boldsymbol{x}^{n}+\left(w_{2}-w_{1}\right) \Delta t \boldsymbol{L}^{n} \boldsymbol{x}^{n}+w_{1} \Delta t \boldsymbol{L}^{n} \tilde{\boldsymbol{x}}+w_{2} \Delta t \boldsymbol{g}^{n}+\left(w_{2} \Delta t\right)^{\frac{1}{2}} \boldsymbol{K}^{n} \boldsymbol{W}_{1}^{n},
$$

and the corrector evaluates the solution at time $(n+1) \Delta t$,

$$
\begin{aligned}
\boldsymbol{x}^{n+1} & =\boldsymbol{x}^{n}+\left(1-w_{3}-w_{4}\right) \Delta t \boldsymbol{L}^{n} \boldsymbol{x}^{n}+w_{3} \Delta t \boldsymbol{L}^{n} \tilde{\boldsymbol{x}}+w_{4} \Delta t \boldsymbol{L}^{n} \boldsymbol{x}^{n+1} \\
& +w_{5} \Delta t\left(\widetilde{\boldsymbol{L}}-\boldsymbol{L}^{n}\right) \tilde{\boldsymbol{x}}+w_{5} \Delta t \tilde{\boldsymbol{g}}+\left(1-w_{5}\right) \Delta t \boldsymbol{g}^{n} \\
& +\Delta t^{\frac{1}{2}}\left[\left(1-w_{6}\right) \boldsymbol{I}+w_{6} \widetilde{\boldsymbol{M}}\left(\boldsymbol{M}^{n}\right)^{-1}\right] \boldsymbol{K}^{n}\left[w_{2}^{\frac{1}{2}} \boldsymbol{W}_{1}^{n}+\left(1-w_{2}\right)^{\frac{1}{2}} \boldsymbol{W}_{2}^{n}\right] .
\end{aligned}
$$

The handling of the multiplicative noise term is inspired by the so-called kinetic interpretation of the stochastic integral [36 and the well-known Fixman method for Brownian dynamics 66. In the above discretization, the standard normal variates $\boldsymbol{W}_{1}^{n}$ correspond to the increment of the underlying Wiener processes over the time interval $w_{2} \Delta t$, $\mathcal{B}\left(n \Delta t+w_{2} \Delta t\right)-\mathcal{B}(n \Delta t)=\left(w_{2} \Delta t\right)^{\frac{1}{2}} \boldsymbol{W}_{1}^{n}$, while the normal variates $\boldsymbol{W}_{2}^{n}$ correspond to the independent increment over the remainder of the time step, $\mathcal{B}((n+1) \Delta t)-\mathcal{B}\left(n \Delta t+w_{2} \Delta t\right)=\left(\left(1-w_{2}\right) \Delta t\right)^{\frac{1}{2}} \boldsymbol{W}_{2}^{n}$.

\section{Additive Noise}

For additive noise, $\boldsymbol{K}(\boldsymbol{x}) \equiv \boldsymbol{K}$, we would like to achieve second-order weak accuracy. A second-order integrator that uses derivatives is provided by the weak Taylor series (16), in indicial notation with the implied summation notation,

$$
\begin{aligned}
x_{\alpha}(t+\Delta t) & =x_{\alpha}(t)+\Delta t L_{\alpha \beta}^{n} x_{\beta}^{n}+\Delta t g_{\alpha}^{n}+K_{\alpha \beta} \Delta \mathcal{B}_{\beta} \\
& +\left(\frac{\Delta t^{2}}{2} L_{\gamma \epsilon}^{n} x_{\epsilon}+\frac{\Delta t^{2}}{2} g_{\gamma}^{n}+\frac{\Delta t}{2} K_{\gamma \epsilon} \Delta \mathcal{B}_{\epsilon}\right)\left(x_{\beta} \partial_{\gamma} L_{\alpha \beta}^{n}+L_{\alpha \gamma}^{n}+\partial_{\gamma} g_{\alpha}^{n}\right) \\
& +\frac{\Delta t^{2}}{4} K_{\gamma \epsilon} K_{\delta \epsilon}\left(x_{\beta} \partial_{\gamma} \partial_{\delta} L_{\alpha \beta}^{n}+2 \partial_{\gamma} L_{\alpha \delta}^{n}+\partial_{\gamma} \partial_{\delta} g_{\alpha}^{n}\right)+O\left(\Delta t^{\frac{5}{2}}\right),
\end{aligned}
$$

where $\Delta \mathcal{B}=\mathcal{B}((n+1) \Delta t)-\mathcal{B}(n \Delta t)$ is the Wiener increment, in law, $\Delta \mathcal{B}=(\Delta t)^{1 / 2} \boldsymbol{W}$ where $\boldsymbol{W}$ is a collection of i.i.d. standard normal random variables.

To prove second-order order weak accuracy for the derivative-free Runge-Kutta scheme, we need to match the first 5 moments of the numerical increment $\Delta_{\alpha}^{n}=x_{\alpha}^{n+1}-x_{\alpha}^{n}$ to the moments of the true increment $\Delta_{\alpha}(n \Delta t)=$ $x_{\alpha}((n+1) \Delta t)-x_{\alpha}(n \Delta t)$, up to order $\Delta t^{2}$ [51]. The increment of the RK method (A1 A2) is (after recursively substituting in for $\boldsymbol{x}^{n+1}$, and Taylor expanding certain terms)

$$
\begin{aligned}
x_{\alpha}^{n+1} & =x_{\alpha}^{n}+\Delta t L_{\alpha \beta}^{n} x_{\beta}^{n}+\Delta t g_{\alpha}^{n}+\Delta t^{\frac{1}{2}} K_{\alpha \beta}\left[w_{2}^{\frac{1}{2}}\left(W_{1}^{n}\right)_{\beta}+\left(1-w_{2}\right)^{\frac{1}{2}}\left(W_{2}^{n}\right)_{\beta}\right] \\
+ & \left(\Delta t^{2} L_{\gamma \epsilon}^{n} x_{\epsilon}^{n}+\Delta t^{2} g_{\gamma}^{n}\right)\left[w_{2} w_{5} x_{\beta}^{n} \partial_{\gamma} L_{\alpha \beta}^{n}+\left(w_{2} w_{3}+w_{4}\right) L_{\alpha \gamma}^{n}+w_{2} w_{5} \partial_{\gamma} g_{\alpha}^{n}\right] \\
+ & \Delta t^{3 / 2} K_{\gamma \epsilon}\left[w_{5} w_{2}^{\frac{1}{2}} x_{\beta}\left(\partial_{\gamma} L_{\alpha \beta}^{n}\right)\left(W_{1}^{n}\right)_{\epsilon}+\left(\left(w_{3}+w_{4}\right) w_{2}^{\frac{1}{2}}\left(W_{1}^{n}\right)_{\epsilon}+w_{4}\left(1-w_{2}\right)^{\frac{1}{2}}\left(W_{2}^{n}\right)_{\epsilon}\right) L_{\alpha \gamma}^{n}+w_{5} w_{2}^{\frac{1}{2}}\left(\partial_{\gamma} g_{\alpha}^{n}\right)\left(W_{1}^{n}\right)_{\epsilon}\right] \\
+ & \frac{w_{2} w_{5}}{2} \Delta t^{2} K_{\gamma \epsilon} K_{\delta \epsilon}\left(x_{\beta} \partial_{\gamma} \partial_{\delta} L_{\alpha \beta}^{n}+2 \partial_{\gamma} L_{\alpha \delta}^{n}+\partial_{\gamma} \partial_{\delta} g_{\alpha}^{n}\right)+O\left(\Delta t^{5 / 2}\right)
\end{aligned}
$$

Comparing the first moments of the increments, $E\left(\Delta_{\alpha}^{n}\right)-E\left(\Delta_{\alpha}(n \Delta t)\right)=O\left(\Delta t^{3}\right)$ if $w_{2} w_{5}=\frac{1}{2}$ and $w_{2} w_{3}+w_{4}=\frac{1}{2}$. The difference in the second moments is also $O\left(\Delta t^{3}\right)$ under the same conditions, as is the difference in the third and fourth moments. The fifth moments are already of $O\left(\Delta t^{3}\right)$. 


\section{Multiplicative Noise}

For multiplicative noise, we only aim to achieve first-order weak accuracy. For this, we need to match the first three moments of the increment up to first order in $\Delta t$ [51. With a variable $\boldsymbol{K}(\boldsymbol{x})$, the difference in the first moments obtains additional terms

$$
\left(\alpha-w_{2} w_{6}\right) \Delta t \partial_{\gamma}\left(K_{\alpha \beta} K_{\gamma \beta}^{n}\right)+O\left(\Delta t^{2}\right)
$$

Therefore, if we have $w_{2} w_{6}=\alpha$, then our method will be consistent with the Ito SDE and reproduce the correct thermal drift without explicitly evaluating $\partial_{\boldsymbol{x}} \cdot \boldsymbol{M}(\boldsymbol{x})$. Note however that this type of "Fixman" approach requires solving one more linear system per time step because of the appearance of $\left(\boldsymbol{M}^{n}\right)^{-1}$ in $(\mathrm{A} 2)$.

Despite recent progress in mathematical understanding [67, multiplicative noise has unclear physical relevance. Fluctuating hydrodynamics is a coarse-grained model in which the variables are averages over many microscopic degrees of freedom and therefore the fluctuations are weak [48, 49, 68. To leading order in the magnitude of the fluctuations, the equations are linear and the noise can be considered additive (though potentially time-dependent). We speculate that multiplicative noise is a very weak perturbation and its effects on the larger scales of the flow, if any, can be sufficiently accurately captured by low-order temporal integrators such as A1 A2 with the condition $w_{2} w_{6}=\alpha$.

\section{Appendix B: L-Stable Scheme}

In typical fluctuating hydrodynamics applications, for explicit schemes the time step is severely limited not by advection but by momentum or heat diffusion, notably, by viscous dissipation. For purely dissipative linear equations, implicit handling of momentum diffusion can yield $A$-stable schemes such as the implicit midpoint scheme (22). This allows the use of much larger time step size $\Delta t$, at least in principle. If one is interested in steady-state fluctuations, the implicit midpoint scheme [22] gives the correct spectrum of fluctuations for any $\Delta t$ (see the Appendix in Ref. [25] for a discussion of how to choose a suitable $\Delta t$ ).

However, for time dependent linear problems, only an exponential integrator can reproduce the correct dynamics for all modes (wavenumbers) for all time step sizes. The implicit midpoint rule provides a notably bad approximation to the exponential decay of correlations for large $\Delta t$, since the Pade $(1,1)$ rational approximation to the exponential (23) $\exp (-x) \approx(1-x / 2) /(1+x / 2)$ tends to -1 for $x \gg 1$ instead of decaying to zero. This leads to oscillatory dynamics for the modes that are under-resolved by the large time step size, i.e., for the thermal fluctuations at large wavenumbers. A much better approximation to $\exp (-x)$ is provided by rational approximations that decay to zero as $x \rightarrow \infty$. In numerical analysis jargon this means handling the diffusive fluxes using an $L$-stable numerical method.

Let us consider the choice of weights in the general scheme 25 that yield a scheme that is weakly second-order accurate and $L$-stable in the implicit part of the dynamics. From the conditions for second-order accuracy 26 we obtain

$$
w_{3}=\frac{\frac{1}{2}-w_{4}}{w_{2}}, \quad w_{5}=\frac{1}{2 w_{2}},
$$

and from the condition of $L$ stability we obtain

$$
w_{1}=\frac{\frac{1}{2}-w_{4}}{1-w_{4}}
$$

which gives the following rational approximation to the exponential decay of the dynamics,

$$
\exp (-x) \approx \frac{\left(1-2 w_{4}+2 w_{4}^{2}\right) x-2\left(1-w_{4}\right)}{\left(w_{4} x+1\right)\left[\left(2 w_{4}-1\right) x-2\left(1-w_{4}\right)\right]}
$$

A reasonable choice of $w_{4}$ can be taken to be the one that minimizes the mismatch between the coefficient in front of $x^{3}$ in the Taylor series expansion of the left and right hand sides [69], giving $w_{4}=1 \pm \sqrt{2} / 2$. In Fig. 6] we compare the two rational approximations by choosing the plus or minus sign. In the deterministic literature the choice of the minus sign has been favored [50, 69, however, we recommend the plus sign,

$$
w_{4}=1+\frac{\sqrt{2}}{2}
$$




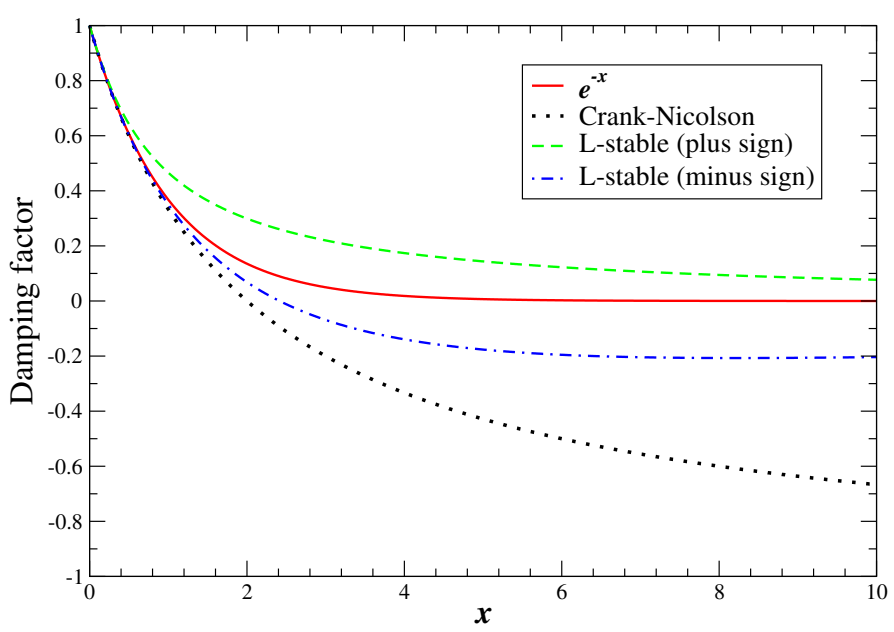

Figure 6: Comparison of $\exp (-x)$ with three rational approximations. The approximation in the Crank-Nicolson scheme 23) does not decay to zero for large $x$. The approximation (B1) decays to zero for $w_{4}=1 \pm \sqrt{2} / 2$, however, only the positive sign gives a strictly positive approximation.

because this gives a strictly positive approximation to the exponential decay of correlations instead of oscillatory behavior for the under-resolved modes (large $x$ ).

We are still left with the choice of $w_{2}$, where the two common choices for $w_{2}$ would be a mid-point, $w_{2}=1 / 2$, or an end-point, $w_{2}=1$, predictor stage. From the discussion in Section IIID 1 we know that when all terms are handled explicitly $(\boldsymbol{L}=\mathbf{0})$, the choice $w_{2}=1 / 2$ gives third-order accuracy for the static covariance for linear problems. It can also be shown that this choice leads to third-order accuracy of the static covariances in the linearized setting if all terms are discretized implicitly $(\boldsymbol{g}(\boldsymbol{x})=\mathbf{0})$. This suggests that a better, even if not unique, choice, is to take $w_{2}=1 / 2$, giving our preferred choice of weights for an $L$-stable predictor-corrector scheme,

$$
w_{1}=w_{4}=1+\frac{\sqrt{2}}{2}, w_{2}=\frac{1}{2}, w_{3}=-(1+\sqrt{2}), w_{5}=1 .
$$

In the linearized setting, this $L$-stable scheme gives second-order accurate covariances for small time step sizes; however, it does not produce the correct spectrum for the fluctuations for large time step sizes, unlike the implicit midpoint scheme (22). In particular, for large $\Delta t$ the $L$-stable scheme strongly damps the magnitude of the fluctuations of the fast (small wavelength or large wavenumber) modes. Therefore, if static covariances are the quantity of interest, the implicit midpoint rule should be used instead.

\section{Appendix C: Approximate Projection Methods}

Here we consider a generalization of the projected Euler-Maruyama scheme (37),

$$
\boldsymbol{v}^{n+1}=\widetilde{\mathbb{P}}\left[\boldsymbol{v}^{n}+\nu \Delta t \boldsymbol{L}_{\boldsymbol{v}} \boldsymbol{v}^{n}+(2 \nu \Delta t)^{\frac{1}{2}} \boldsymbol{D}_{\boldsymbol{w}} \boldsymbol{W}_{\boldsymbol{v}}^{n}\right],
$$

where $\widetilde{\mathbb{P}}$ an approximation to the discrete projection $\mathbb{P}$, for example, $\mathbb{P}=\boldsymbol{I}-\boldsymbol{G} \boldsymbol{L}_{p}^{-1} \boldsymbol{D}$. Here $\boldsymbol{L}_{p}$ is a discrete pressure Laplacian operator that may, in general, be different from $\boldsymbol{L}_{s}=\boldsymbol{D} \boldsymbol{G}$. For example, with spatial discretizations of the incompressible (Navier-)Stokes equations that use cell-centered velocities, $\boldsymbol{L}_{s}$ possesses a non-trivial nullspace and the corresponding exact projection methods (in which $\widetilde{\mathbb{P}}=\mathbb{P}$ ) suffer from the so-called checkerboard instability. Approximate projection methods have been developed to overcome these difficulties of exact cell-centered projection methods [61. One of the simplest approximate projection methods is (C1) with $\boldsymbol{L}_{p}$ being the standard second-order Laplacian stencil [62]. For the staggered-grid spatial discretization we employ, however, it is straightforward to invert $\boldsymbol{L}_{s}$ and approximate projection methods are not used in practice.

The steady-state covariance of the iteration (C1) should be a consistent approximation to the continuum result (34). Specifically, we ask that to leading order in the time step size

$$
\boldsymbol{C}_{\boldsymbol{v}}=\left\langle\boldsymbol{v}^{n+1}\left(\boldsymbol{v}^{n+1}\right)^{\star}\right\rangle=\left\langle\boldsymbol{v}^{n}\left(\boldsymbol{v}^{n}\right)^{\star}\right\rangle=\mathbb{P}+\Delta t \Delta \boldsymbol{C}_{\boldsymbol{v}}+O\left(\Delta t^{2}\right) .
$$


Substituting (C1) in this condition and equating the leading-order terms we obtain the condition

$$
\widetilde{\mathbb{P}} \widetilde{\mathbb{P}}^{\star}=\mathbb{P} .
$$

This condition is satisfied for exact projection methods, $\widetilde{\mathbb{P}}=\mathbb{P}$, but not for approximate projection methods, $\widetilde{\mathbb{P}} \neq \mathbb{P}$. Assuming the initial condition is discretely divergence-free, for exact projection (C1) is equivalent to (37).

\section{Appendix D: Mode Analysis}

It is instructive to describe a framework for analyzing schemes such as (39), following the mode analysis used to study splitting errors in projection methods in the deterministic context 70, 71]. This analysis can in principle produce explicit expressions for the spectrum of velocity fluctuations for the types of schemes we consider here. It also illustrates clearly the role of the pressure and, in particular, the difficulties with applying semi-implicit projection (splitting) methods in the context of the fluctuating Navier-Stokes equations.

A mode of the spatially-discretized unforced time-dependent (creeping) Stokes flow equation

$$
\partial_{t} \boldsymbol{v}+\boldsymbol{G} \boldsymbol{\pi}=\nu \boldsymbol{L}_{\boldsymbol{v}} \boldsymbol{v}, \text { s.t. } \boldsymbol{D} \boldsymbol{v}=0
$$

is an exponentially-decaying solution of the form

$$
\boldsymbol{v}(t)=\boldsymbol{v}_{0} e^{-\sigma t} \text { and } \boldsymbol{\pi}(t)=\nu \boldsymbol{\pi}_{0} e^{-\sigma t} .
$$

Here $\sigma \geq 0$ is the decay rate associated with the spatial mode $\left\{\boldsymbol{v}_{0}, \boldsymbol{\pi}_{0}\right\}$, which is a normalized solution to the eigen-problem

$$
\left(\boldsymbol{L}_{\boldsymbol{v}}+\nu^{-1} \sigma \boldsymbol{I}\right) \boldsymbol{v}_{0}+\boldsymbol{G} \boldsymbol{\pi}_{0}=0 \text { and } \boldsymbol{D} \boldsymbol{v}_{0}=0 .
$$

These modes diagonalize the creeping Stokes flow dynamics and form a complete orthonormal basis for the space of divergence-free velocity fields. This can be seen by eliminating the pressure to obtain the classical eigenvalue problem in the subspace of discretely divergence-free velocity fields

$$
\left[\boldsymbol{L}_{\boldsymbol{v}}-\boldsymbol{L}_{\boldsymbol{v}}^{-1} \boldsymbol{G}\left(\boldsymbol{D} \boldsymbol{L}_{\boldsymbol{v}}^{-1} \boldsymbol{G}\right)^{-1} \boldsymbol{D} \boldsymbol{L}_{\boldsymbol{v}}^{-1}\right] \boldsymbol{v}_{0}=-\nu^{-1} \sigma \boldsymbol{v}_{0}
$$

In the presence of a stochastic forcing, we can express any solution in a basis formed by the modes $\left\{\boldsymbol{v}_{0}^{1}, \boldsymbol{v}_{0}^{2}, \ldots\right\}$,

$$
\boldsymbol{v}(t)=\sum_{k} v_{k}(t) \boldsymbol{v}_{0}^{k}
$$

where the mode amplitudes $v_{k}(t)$ are scalar stochastic processes. The stochastic forcing $(2 \nu)^{\frac{1}{2}} \boldsymbol{D}_{\boldsymbol{w}} \mathcal{W}_{\boldsymbol{v}}(t)$ in the momentum equation can be projected onto $\boldsymbol{v}_{0}^{k}$ to obtain the amplitude of the stochastic forcing for mode $k$,

$$
w_{k}(t)=(2 \nu)^{\frac{1}{2}}\left(\boldsymbol{v}_{0}^{k}\right)^{\star}\left(\boldsymbol{D}_{\boldsymbol{w}} \mathcal{W}_{\boldsymbol{v}}(t)\right),
$$

which is a scalar white-noise process with covariance

$$
\left\langle w_{k}(t) w_{k}^{\star}\left(t^{\prime}\right)\right\rangle=2 \nu\left(\boldsymbol{v}_{0}^{k}\right)^{\star}\left[\boldsymbol{D}_{\boldsymbol{w}}\left\langle\mathcal{W}_{\boldsymbol{v}}(t) \mathcal{W}_{\boldsymbol{v}}^{\star}\left(t^{\prime}\right)\right\rangle \boldsymbol{D}_{\boldsymbol{w}}^{\star}\right]\left(\boldsymbol{v}_{0}^{k}\right)=-2 \nu\left(\boldsymbol{v}_{0}^{k}\right)^{\star} \boldsymbol{L}_{\boldsymbol{v}}\left(\boldsymbol{v}_{0}^{k}\right) \delta\left(t-t^{\prime}\right),
$$

where we made use of the discrete fluctuation-dissipation balance between the viscous dissipation and the stochastic forcing, $\boldsymbol{L}_{\boldsymbol{v}}=-\boldsymbol{D}_{\boldsymbol{w}}\left(\boldsymbol{D}_{\boldsymbol{w}}\right)^{\star}$. From (D2) we can express

$$
-2 \nu\left(\boldsymbol{v}_{0}^{k}\right)^{\star} \boldsymbol{L}_{\boldsymbol{v}}\left(\boldsymbol{v}_{0}^{k}\right)=-2 \nu\left(\boldsymbol{v}_{0}^{k}\right)^{\star}\left(\boldsymbol{G} \boldsymbol{\pi}_{0}-\nu^{-1} \sigma_{k} \boldsymbol{v}_{0}^{k}\right)=2 \nu\left(\boldsymbol{D} \boldsymbol{v}_{0}^{k}\right)^{\star} \boldsymbol{\pi}_{0}+2 \sigma_{k}\left\|\boldsymbol{v}_{0}^{k}\right\|^{2}=2 \sigma_{k},
$$

where we again made use of the duality relation $G=-D^{\star}$. This simple calculation shows that in the mode representation the linearized fluctuating Navier-Stokes equation becomes a collection of decoupled scalar Langevin equations driven by standard Wiener processes,

$$
\frac{d v_{k}}{d t}=-\sigma_{k} v_{k}+\left(2 \sigma_{k}\right)^{\frac{1}{2}} \mathcal{W}_{v}(t)
$$


The fluctuation-dissipation balance is most clearly revealed in this representation.

Temporal discretizations can be analyzed by projecting the numerical solution onto a set of discrete modes. For the implicit midpoint discretization (39), the modes are decaying solutions of the form

$$
\boldsymbol{v}_{k}^{n+1}=\boldsymbol{v}_{k}^{n} e^{-\tilde{\sigma}_{k} \Delta t} \text { and } \boldsymbol{\pi}_{k}^{n+\frac{1}{2}}=\nu \boldsymbol{\pi}_{k}^{n} e^{-\tilde{\sigma}_{k} \Delta t / 2},
$$

where $\tilde{\sigma}_{k} \approx \sigma_{k}$ is the numerical decay rate. The spatial structure of the mode $\left\{\boldsymbol{v}_{k}^{n}, \boldsymbol{\pi}_{k}^{n}\right\}$ is the solution to the discrete eigen-problem

$$
\left[\boldsymbol{L}_{\boldsymbol{v}}+\nu^{-1}\left(\frac{2}{e^{-\tilde{\sigma}_{k} \Delta t}+1}\right)\left(\frac{1-e^{-\tilde{\sigma}_{k} \Delta t}}{\Delta t}\right) \boldsymbol{I}\right] \boldsymbol{v}_{k}^{n}+\left(\frac{2}{e^{-\tilde{\sigma}_{k} \Delta t}+1}\right) e^{-\tilde{\sigma}_{k} \Delta t / 2} \boldsymbol{G} \boldsymbol{\pi}_{k}^{n}=\boldsymbol{v}^{n} .
$$

Comparison to (D2) shows that the spatial modes are the same as for the semi-continuum (D1), and the temporal decay rate is second-order accurate in the time step,

$$
\sigma_{k}=\left(\frac{2}{e^{-\tilde{\sigma}_{k} \Delta t}+1}\right)\left(\frac{1-e^{-\tilde{\sigma}_{k} \Delta t}}{\Delta t}\right)=\tilde{\sigma}_{k}\left(1-\frac{\tilde{\sigma}_{k}^{2} \Delta t^{2}}{12}\right)+O\left(\Delta t^{3}\right) .
$$

When the stochastic forcing is included, the discrete velocity can be represented in the basis formed by the discrete modes just as we did above for the time-continuous equations. In the mode representation the scheme 39 is seen to be nothing more than the implicit midpoint method (22) applied to the system of decoupled SDEs (D3).

The mode analysis reveals that semi-implicit projection (splitting) methods have a significant shortcoming not seen for explicit methods. A Crank-Nicolson projection method for (D1) consists of first solving the following linear system for the velocity $\boldsymbol{v}^{n+1}$ with a time-lagged pressure [72,

$$
\left(\boldsymbol{I}-\frac{\nu \Delta t}{2}\right) \tilde{\boldsymbol{v}}^{n+1}+\Delta t \boldsymbol{G} \boldsymbol{\pi}^{n-\frac{1}{2}}=\left(\boldsymbol{I}+\frac{\nu \Delta t}{2}\right) \boldsymbol{v}^{n}
$$

and then projecting the intermediate velocity $\tilde{\boldsymbol{v}}^{n+1}$ to enforce the divergence-free constraint, $\boldsymbol{v}^{n+1}=\mathbb{P} \tilde{\boldsymbol{v}}^{n+1}$, by solving a linear system for the pressure correction

$$
\boldsymbol{v}^{n+1}=\tilde{\boldsymbol{v}}^{n+1}-\Delta t \boldsymbol{G} \Delta \boldsymbol{\pi}^{n} \text {, s.t. } \boldsymbol{D} \boldsymbol{v}^{n+1}=0 .
$$

Repeating the discrete mode calculation reveals that the spatial modes for the above temporal discretization are not the same as for the semi-continuum (D1), specifically, the gradient of pressure term in (D2) is modified by a term involving the Laplacian $\boldsymbol{L}_{\boldsymbol{v}}$. For periodic systems the discrete gradient and vector Laplacian commute, $\boldsymbol{L}_{\boldsymbol{v}} \boldsymbol{G}=\boldsymbol{G} \boldsymbol{L}_{s}$, and modes have the correct spatial structure. However, for non-periodic systems the splitting of the pressure and velocity equations introduces a commutator error that leads to the appearance of "spurious" or "parasitic" modes [71. For deterministic solutions and moderate time step sizes, spatio-temporal smoothness of the solution usually makes these commutator errors acceptably small. In the stochastic context, however, all modes are stochastically forced and have a non-negligible amplitude, including the parasitic modes. For this reason, we chose to use (39) and solve a coupled Stokes linear system for both pressure and velocity, and only use the projection method as a preconditioner for the required Krylov solver [63. We emphasize again that for purely explicit time stepping scheme the spatial structure of the modes is preserved and projection methods can be used in the stochastic setting as well.

[1] C. W. Gardiner. Handbook of stochastic methods: for physics, chemistry 8 the natural sciences, volume Vol. 13 of Series in synergetics. Springer, third edition, 2003.

[2] H.C. Öttinger. Beyond equilibrium thermodynamics. Wiley Online Library, 2005.

[3] L. Bocquet and E. Charlaix. Nanofluidics, from bulk to interfaces. Chemical Society Reviews, 39(3):1073-1095, 2010.

[4] G. Hu and D. Li. Multiscale phenomena in microfluidics and nanofluidics. Chemical Engineering Science, 62(13):3443-3454, 2007.

[5] L. Wang and M. Quintard. Nanofluids of the future. Advances in Transport Phenomena, pages 179-243, 2009.

[6] F. Detcheverry and L. Bocquet. Thermal fluctuations in nanofluidic transport. Phys. Rev. Lett., 109:024501, 2012.

[7] A. Vailati, R. Cerbino, S. Mazzoni, C. J. Takacs, D. S. Cannell, and M. Giglio. Fractal fronts of diffusion in microgravity. Nature Communications, 2:290, 2011.

[8] A. Donev, A. L. Garcia, Anton de la Fuente, and J. B. Bell. Enhancement of Diffusive Transport by Nonequilibrium Thermal Fluctuations. J. of Statistical Mechanics: Theory and Experiment, 2011:P06014, 2011.

[9] D. Panja. Effects of fluctuations on propagating fronts. Physics Reports, 393(2):87-174, 2004. 
[10] W. Van Saarloos. Front propagation into unstable states. Physics Reports, 386(2-6):29-222, 2003.

[11] R. Delgado-Buscalioni, E. Chacon, and P. Tarazona. Hydrodynamics of nanoscopic capillary waves. Phys. Rev. Lett., 101(10):106102, 2008.

[12] B. Z. Shang, N. K. Voulgarakis, and J.-W. Chu. Fluctuating hydrodynamics for multiscale simulation of inhomogeneous fluids: Mapping all-atom molecular dynamics to capillary waves. J. Chem. Phys., 135:044111, 2011.

[13] M. Moseler and U. Landman. Formation, stability, and breakup of nanojets. Science, 289(5482):1165-1169, 2000.

[14] B. Davidovitch, E. Moro, and H.A. Stone. Spreading of viscous fluid drops on a solid substrate assisted by thermal fluctuations. Phys. Rev. letters, 95(24):244505, 2005.

[15] Y. Hennequin, D. G. A. L. Aarts, J. H. van der Wiel, G. Wegdam, J. Eggers, H. N. W. Lekkerkerker, and D. Bonn. Drop formation by thermal fluctuations at an ultralow surface tension. Phys. Rev. Lett., 97(24):244502, 2006.

[16] L.D. Landau and E.M. Lifshitz. Fluid Mechanics, volume 6 of Course of Theoretical Physics. Pergamon Press, Oxford, England, 1959.

[17] J. M. O. De Zarate and J. V. Sengers. Hydrodynamic fluctuations in fluids and fluid mixtures. Elsevier Science Ltd, 2006.

[18] D. Bedeaux, I. Pagonabarraga, J.M.O. de Zárate, J.V Sengers, and S. Kjelstrup. Mesoscopic non-equilibrium thermodynamics of non-isothermal reaction-diffusion. Phys. Chem. Chem. Phys., 12(39):12780-12793, 2010.

[19] R.V. Kohn, M.G. Reznikoff, and E. Vanden-Eijnden. Magnetic elements at finite temperature and large deviation theory. Journal of nonlinear science, 15(4):223-253, 2005.

[20] J. L. Lebowitz, E. Presutti, and H. Spohn. Microscopic models of hydrodynamic behavior. J. Stat. Phys., 51(5):841-862, 1988.

[21] P. Español. Stochastic differential equations for non-linear hydrodynamics. Physica A, 248(1-2):77-96, 1998.

[22] P. Español, J.G. Anero, and I. Zúñiga. Microscopic derivation of discrete hydrodynamics. J. Chem. Phys., 131:244117, 2009.

[23] B. Leimkuhler and S. Reich. Simulating hamiltonian dynamics, volume 14. Cambridge University Press, 2005.

[24] A. K. Bhattacharjee, G. I. Menon, and R. Adhikari. Fluctuating dynamics of nematic liquid crystals using the stochastic method of lines. J. Chem. Phys., 133:044112, 2010.

[25] F. Balboa Usabiaga, J. B. Bell, R. Delgado-Buscalioni, A. Donev, T. G. Fai, B. E. Griffith, and C. S. Peskin. Staggered Schemes for Incompressible Fluctuating Hydrodynamics. SIAM J. Multiscale Modeling and Simulation, 10(4):1369-1408, 2012.

[26] J.D. Ramshaw and K. Lindenberg. Augmented Langevin description of multiplicative noise and nonlinear dissipation in Hamiltonian systems. J. Stat. Phys, 45(1):295-307, 1986.

[27] P.J. Morrison. Hamiltonian description of the ideal fluid. Rev. Mod. Phys., 70(2):467, 1998.

[28] T.J. Bridges and S. Reich. Numerical methods for hamiltonian PDEs. Journal of Physics A: Mathematical and General, 39:5287, 2006.

[29] P. Español and I. Zúñiga. On the definition of discrete hydrodynamic variables. J. Chem. Phys, 131:164106, 2009.

[30] J. A. de la Torre and P. Español. Coarse-graining Brownian motion: From particles to a discrete diffusion equation. J. Chem. Phys, 135:114103, 2011.

[31] A. Donev, A. L. Garcia, Anton de la Fuente, and J. B. Bell. Diffusive Transport by Thermal Velocity Fluctuations. Phys. Rev. Lett., 106(20):204501, 2011.

[32] A. Rocco, J. Casademunt, U. Ebert, and W. Van Saarloos. Diffusion coefficient of propagating fronts with multiplicative noise. Physical Review E, 65(1):012102, 2001.

[33] J.B. Bell, A.L. Garcia, and S.A. Williams. Numerical methods for the stochastic Landau-Lifshitz Navier-Stokes equations. Phys. Rev. E, 76:016708, 2007.

[34] P. Español. Statistical mechanics of coarse-graining. Novel Methods in Soft Matter Simulations, pages 2256-2256, 2004.

[35] H. Grabert. Projection operator techniques in nonequilibrium statistical mechanics. Springer-Verlag, 1982.

[36] M. Hütter and H.C. Öttinger. Fluctuation-dissipation theorem, kinetic stochastic integral and efficient simulations. J. Chem. Soc., Faraday Trans., 94(10):1403-1405, 1998.

[37] J. B. Bell, J. Foo, and A. L. Garcia. Algorithm refinement for the stochastic Burgers equation. J. Comput. Phys., $223(1): 451-468,2007$.

[38] M. Hairer and J. Voss. Approximations to the Stochastic Burgers equation. Journal of NonLinear Science, pages 1-24, 2010.

[39] H.C. Öttinger. Constraints in nonequilibrium thermodynamics: General framework and application to multicomponent diffusion. J. Chem. Phys., 130:114904, 2009.

[40] A. Majda and I. Tomofeyev. Statistical mechanics for truncations of the Burgers-Hopf equation: a model for intrinsic stochastic behavior with scaling. Milan Journal of Mathematics, 70(1):39-96, 2002.

[41] A. Donev, E. Vanden-Eijnden, A. L. Garcia, and J. B. Bell. On the Accuracy of Explicit Finite-Volume Schemes for Fluctuating Hydrodynamics. CAMCOS, 5(2):149-197, 2010.

[42] Y. Morinishi, T.S. Lund, O.V. Vasilyev, and P. Moin. Fully conservative higher order finite difference schemes for incompressible flow. J. Comp. Phys., 143(1):90-124, 1998.

[43] D.F. Anderson and J.C. Mattingly. A weak trapezoidal method for a class of stochastic differential equations. Communications in Mathematical Sciences, 9(1):301-318, 2011.

[44] C.M. Mora. Weak exponential schemes for stochastic differential equations with additive noise. IMA journal of numerical analysis, 25(3):486-506, 2005.

[45] G.J. Lord and A. Tambue. Stochastic exponential integrators for finite element discretization of SPDEs for multiplicative and additive noise. Arxiv preprint arXiv:1103.1986, 2011. 
[46] H. De la Cruz Cancino, R.J. Biscay, J.C. Jimenez, F. Carbonell, and T. Ozaki. High order local linearization methods: An approach for constructing A-stable explicit schemes for stochastic differential equations with additive noise. BIT Numerical Mathematics, 50(3):509-539, 2010.

[47] P. J. Atzberger, P. R. Kramer, and C. S. Peskin. A stochastic immersed boundary method for fluid-structure dynamics at microscopic length scales. J. Comp. Phys., 224:1255-1292, 2007.

[48] E. Buckwar and R. Winkler. Multistep methods for SDEs and their application to problems with small noise. SIAM journal on numerical analysis, pages 779-803, 2006.

[49] H.D. Ceniceros and G.O. Mohler. A practical splitting method for stiff sdes with applications to problems with small noise. Multiscale Modeling and Simulation, 6(1):212, 2007.

[50] U.M. Ascher, S.J. Ruuth, and B.T.R. Wetton. Implicit-explicit methods for time-dependent partial differential equations. SIAM Journal on Numerical Analysis, pages 797-823, 1995.

[51] G.N. Milstein and M.V. Tretyakov. Stochastic numerics for mathematical physics. Springer, 2004.

[52] L. Machiels and M.O. Deville. Numerical simulation of randomly forced turbulent flows. J. Comp. Phys., 145(1):246-279, 1998.

[53] N. Bou-Rabee and E. Vanden-Eijnden. Pathwise accuracy and ergodicity of metropolized integrators for SDEs. Communications on Pure and Applied Mathematics, 63(5):655-696, 2010.

[54] J.C. Mattingly, A.M. Stuart, and M.V. Tretyakov. Convergence of Numerical Time-Averaging and Stationary Measures via Poisson Equations. SIAM Journal on Numerical Analysis, 48:552, 2010.

[55] P. J. Atzberger. Spatially Adaptive Stochastic Numerical Methods for Intrinsic Fluctuations in Reaction-Diffusion Systems. J. Comp. Phys., 229(9):3474 - 3501, 2010.

[56] S. Gottlieb and C. Shu. Total variation diminishing Runge-Kutta schemes. Mathematics of Computation, 67(221):73-85, 1998.

[57] K. Debrabant. Runge-Kutta methods for third order weak approximation of SDEs with multidimensional additive noise. BIT, 50(3):541-558, 2010.

[58] E. Vanden-Eijnden and G. Ciccotti. Second-order integrators for Langevin equations with holonomic constraints. Chemical physics letters, 429(1-3):310-316, 2006.

[59] G. Ciccotti, T. Lelièvre, and E. Vanden-Eijnden. Projection of diffusions on submanifolds: Application to mean force computation. Communications on Pure and Applied Mathematics, 61(3):371-408, 2008.

[60] F.H. Harlow and J.E. Welch. Numerical calculation of time-dependent viscous incompressible flow of fluids with free surfaces. Physics of Fluids, 8:2182-2189, 1965.

[61] A. S. Almgren, J. B. Bell, and W. G. Szymczak. A numerical method for the incompressible Navier-Stokes equations based on an approximate projection. SIAM J. Sci. Comput., 17(2):358-369, March 1996.

[62] A.S. Almgren, J.B. Bell, and W.Y. Crutchfield. Approximate projection methods: Part I. Inviscid analysis. SIAM Journal on Scientific Computing, 22:1139, 2000.

[63] B.E. Griffith. An accurate and efficient method for the incompressible Navier-Stokes equations using the projection method as a preconditioner. J. Comp. Phys., 228(20):7565-7595, 2009.

[64] B.E. Griffith, R.D. Hornung, D.M. McQueen, and C.S. Peskin. An adaptive, formally second order accurate version of the immersed boundary method. J. Comput. Phys., 223(1):10-49, 2007. Software available at http://ibamr.googlecode.com

[65] J. A. Pérez. Convergence of numerical schemes in the total variation sense. PhD thesis, New York University, Department of Computer Science, 2006.

[66] M. Fixman. Simulation of polymer dynamics. I. General theory. J. Chem. Phys., 69:1527, 1978.

[67] M. Hairer and H. Weber. Rough Burgers-like equations with multiplicative noise. Probability Theory and Related Fields, pages $1-56,2010$.

[68] G.N. Milstein and M.V. Tretyakov. Numerical methods in the weak sense for stochastic differential equations with small noise. SIAM journal on numerical analysis, pages 2142-2167, 1997.

[69] E.H. Twizell, A.B. Gumel, and M.A. Arigu. Second-order, $L_{0}$-stable methods for the heat equation with time-dependent boundary conditions. Advances in Computational Mathematics, 6(1):333-352, 1996.

[70] W. E and J.G. Liu. Projection method III: Spatial discretization on the staggered grid. Mathematics of Computation, 71(237):27-48, 2002.

[71] W. E and J.G. Liu. Gauge method for viscous incompressible flows. Commun. Math. Sci, 1(2):317-332, 2003.

[72] J. B. Bell, P. Colella, and H. M. Glaz. A second order projection method for the incompressible Navier-Stokes equations. J. Comp. Phys., 85(2):257-283, 1989. 